\title{
Severe deficiency of voltage-gated sodium channel Nav1.2 elevates neuronal excitability in adult mice
}

\author{
Short title: Nav1.2 deficiency and neuronal hyperexcitability
}

Jingliang Zhang ${ }^{1,2 *}$, Xiaoling Chen ${ }^{1,2,3 *}$, Muriel Eaton ${ }^{1,2}$, Shirong Lai ${ }^{1,2}$, Anthony Park ${ }^{1,2}$, Talha S. Ahmad ${ }^{1,2}$, Jiaxiang $\mathrm{Wu}^{1,2}$, Zhixiong $\mathrm{Ma}^{1,2}$, Zhefu Que ${ }^{1,2}$, Ji Hea Lee ${ }^{1,2}$, Tiange Xiao $^{1,2}$, Yuansong $\mathrm{Li}^{1,2}$, Yujia Wang ${ }^{1,2}$, Maria I. Olivero-Acosta ${ }^{1,2}$, James A. Schaber ${ }^{4}$, Krishna Jayant ${ }^{2,3}$, Zhuo Huang ${ }^{5}$, Nadia A. Lanman ${ }^{6,7}$, William C. Skarnes ${ }^{8}$, Yang Yang ${ }^{1,2 \dagger}$

${ }^{1}$ Department of Medicinal Chemistry and Molecular Pharmacology, College of Pharmacy, Purdue University.

${ }^{2}$ Purdue Institute for Integrative Neuroscience, Purdue University.

${ }^{3}$ Weldon School of Biomedical Engineering, Purdue University.

${ }^{4}$ Bioscience Imaging Facility, Bindley Bioscience Center, Purdue University.

${ }^{5}$ Department of Molecular and Cellular Pharmacology, School of Pharmaceutical Sciences, Peking University Health Science Center.

${ }^{6}$ Department of Comparative Pathobiology, Purdue University.

${ }^{7}$ Purdue University Center for Cancer Research, Purdue University.

${ }^{8}$ The Jackson Laboratory for Genomic Medicine.

*These authors contributed equally to this work.

tCorresponding author. Email: yangyang@purdue.edu

\section{Abstract}

Scn2a encodes voltage-gated sodium channel Nav1.2, which mediates neuronal firing. The current paradigm suggests that Nav1.2 gain-of-function variants enhance neuronal excitability resulting in epilepsy, whereas Nav1.2 deficiency impairs neuronal excitability contributing to autism. In this paradigm, however, why about a third of patients with Nav1.2 deficiency still develop seizures remains a mystery. Here we challenge the conventional wisdom, reporting that neuronal excitability is increased with severe Nav1.2 deficiency. Using a unique gene-trap knockout mouse model of Scn2a, we found enhanced intrinsic excitabilities of principal neurons in the cortico-striatal circuit, known to be involved in Scn2a-related seizures. This increased excitability is autonomous, and is reversible by genetic restoration of Scn2a expression in adult mice. Mechanistic investigation reveals a compensatory downregulation of potassium channels including Kv1.1, which could be targeted to alleviate neuronal hyperexcitability. Our unexpected findings may explain Nav1.2 deficiency-related epileptic seizures in humans and provide molecular targets for potential interventions.

KEYWORDS: Voltage-gated sodium channel, Nav1.2, SCN2A/Scn2a gene, gene-trap Kv1.1 


\section{TEASER:}

43 Severe Nav1.2 deficiency results in neuronal hyperexcitability via the compensatory

44 downregulation of potassium channels.

45

\section{HIGHLIGHTS:}

47 1. Severe Nav1.2 deficiency results in enhanced excitability of medium spiny neurons 48 (MSNs) and pyramidal neurons in adult mice;

49 2. Increased neuronal excitability in MSNs is accompanied by elevated voltage threshold;

50 3. Nav1.2 deficiency-related hyperexcitability is reversible with the restoration of Scn2a 51 expression, and is autonomous;

52 4. The expression of the Kv1.1 channel has a compensatory reduction in neurons with 53 Nav1.2 deficiency, and Kv channels openers normalize the neuronal excitability;

54 5. The enhanced excitability in brain slices translates to elevated in vivo firing commonly 55 associated with seizures. 


\section{INTRODUCTION}

Nav1.2 channel, encoded by SCN2A, is a major voltage-gated sodium channel expressed in the central nervous system (CNS) supporting the action potentials (AP) firing (1) (2). Nav1.2 is strongly expressed in the principal neurons of the cortico-striatal circuit, including pyramidal neurons of the medial prefrontal cortex (mPFC) and medium spiny neurons (MSNs) of the caudate-putamen (CPu) in the striatum (3-5). Gain-of-function (GoF) variants of SCN2A are closely associated with epileptic seizures, whereas loss-offunction (LoF) or protein-truncating variants of SCN2A (collectively referred to as Nav1.2 deficiency) are leading genetic causes of autism spectrum disorder (ASD) and intellectual disability (ID) (6-10). The conventional paradigm suggests that GoF variants of SCN2A increase the excitability of principal neurons resulting in epilepsy, whereas Nav1.2 deficiency impairs the excitability of principal neurons leading to ASD (2). However, clinical studies found that a significant portion of patients with Nav1.2 deficiency develop "late-onset" intractable seizures $(11,12)$. As hyperexcitability and hypersynchronization of neuronal firings are suggested as the basis of seizures (13), it is thus intriguing how Nav1.2 deficiency, predicted to reduce neuronal excitability, contributes to epileptic seizures.

To understand Nav1.2 deficiency-related pathophysiology, mouse models were generated. Homozygous Scn2a ${ }^{-/}$knockout mice die perinatally $(14,15)$; Heterozygous Scn2a ${ }^{+/-}$mice (with $\sim 50 \%$ Nav1.2 expression level) survive to adulthood, but the earlier study did not find notable abnormalities in Scn2a ${ }^{+-}$mice (14). More recently, absencelike seizures were reported in adult male Scn2a ${ }^{+/-}$mice (16). It is suggested that the CPu of the striatum and the mPFC of the cortex are key brain regions in which absence seizure-like spike-wave discharges (SWDs) were identified $(16,17)$. Indeed, the corticostriatal circuit is highly involved in ASD as well as seizures, and the excitability of principal neurons in this circuit could strongly influence seizure susceptibility $(18,19)$. Despite these in vivo findings, recordings in brain slices, however, revealed unchanged AP firings and reduced excitatory postsynaptic current in pyramidal neurons of adult Scn2a ${ }^{+/-}$mice $(16,20)$, leaving cellular mechanisms ambiguous.

It is not uncommon that phenotypes observed in hemizygous patients do not manifest in heterozygous mouse models. In fact, it is known that mice are more tolerant than humans to certain gene expression reduction (21). Therefore, the heterozygous knockout with a close to $50 \%$ reduction in Scn2a protein level may not be sufficient to render major phenotypes in mice (21). A more substantial reduction of gene expression could be essential to produce robust phenotypes in the mouse model of Nav1.2 deficiency. Because Scn2a null (100\% knockout) is lethal, we thus generated a novel Nav1.2-deficient mouse model via a gene-trap knockout (gtKO) strategy (22). These mice display many behavioral abnormalities, modeling aspects of phenotypes in humans with SCN2A deficiency (22). Using this unique mouse model, we investigated how severe Nav1.2 deficiency affects neuronal excitabilities of principal neurons in the cortico-striatal circuit. Our results demonstrate a surprising hyperexcitability phenotype in neurons, in which the compensatory downregulation of the potassium channels is likely to be an underlying mechanism. 


\section{RESULTS}

\section{Neurons expressing substantially low Nav1.2 exhibit elevated excitability}

To understand how a severe Nav1.2 deficiency affects the function of neurons, we utilized a gene-trap knockout mouse model of Scn2a. Homozygous Scn2agtKO/gtKo mice (referred to as HOM herein) can survive to adulthood, and have a substantial reduction of Nav1.2 expression ( $25 \%$ of the WT level) (22). Because the gene-trap cassette contains a LacZ element, which is driven by the native Nav1.2 promoter (Figure S1A) (23, 24), we used LacZ-staining as a surrogate to determine the expression and distribution of Nav1.2 in the brain. Our data showed that Scn2a is widely expressed in the mouse brain including the cortex and striatum (Figure S1B), which is consistent with previous studies of Scn2a distribution (3-5).

The CPu is a common node for ASD and seizures, and is one of the major brain regions involved in the Scn2a-related absence-like seizures (16-18). Previous study and our LacZ-staining suggested that Nav1.2 is highly expressed in the CPu. To further confirm these results, we performed Western blot analysis. We found that the heterozygous (HET) Scn2aWT/gtKO mice have $60 \%$ of WT Nav1.2 protein level in the CPu tissues, whereas the homozygous (HOM) Scn2agtKO/gtKO mice have a much lower level at 34\% (Figure S1C). This result is largely consistent with our initial characterization of this mouse model using whole-brain samples (22). To understand how a severe deficiency of Nav1.2 affects neuronal excitability, we performed ex vivo patch-clamp recordings in brain slices from adult Scn2agtKo/gtKo mice. Unexpectedly, we found that the striatal principal medium spiny neurons (MSNs) from Scn2agtKO/gtKo mice were markedly more excitable (Figure 1A-C). The current-injection triggered action potential (AP) number was significantly elevated in MSNs from Scn2agtKo/gtKo mice compared to WT littermates. We also observed depolarized resting membrane potential (RMP) and increased input resistance of these MSNs (Figure 1D, E), which were in line with the increased neuronal excitability. Phase-plane plot analysis showed that the AP waveform in Scn2agtKO/gtKO mice was altered (Figure 1F, G). While rheobase was reduced, interestingly we detected a higher voltage threshold, reduced AP amplitude, elevated fast after-hyperpolarization (fAHP), and increased half-width values in MSNs from Scn2agtKO/gtKo mice (Figure 1H-L). Voltage-dependent conductance can affect neuronal RMP (25), and RMP is known to influence neuronal excitability (26). We thus performed recordings at a fixed membrane potential (MP) to understand whether the altered RMP is a major factor for this observed hyperexcitability of MSNs. However, even at the fixed MP, we were still able to detect the enhanced excitability along with the altered AP waveforms in Scn2agtKO/gtKO mice (Figure S1D-M), suggesting that besides the RMP, other factors are playing essential roles contributing to the neuronal hyperexcitability. Taken together, our data suggest a counterintuitive finding that severe deficiency of Nav1.2 renders an increased (rather than conventionally suggested as decreased) neuronal excitability.

\section{Enhanced excitability is reversible in adult Nav1.2-deficient mice with the restoration of Scn2a expression, and is autonomous}

Scn2agtKo/gtKo mice, generated via a gene-trap strategy, has a built-in genetic "rescue" element for manipulations $(24,27)$. The inserted "tm1a" trapping cassette is flanked with 
Frt sites, which can be removed via a flippase recombinase (Flp) to achieve a "tm1c" allele in a temporally and spatially controlled manner (24) (Figure S1A). This "tm1c" allele is practically a "rescue" allele to restore the expression of the target gene. We performed experiments to restore the Scn2a expression by adeno-associated virus (AAV) delivery of codon-optimized Flp (FlpO), with a goal to determine the reversibility of these enhanced neuronal firings in adult mice. Using a PHP.eB.AAV vector, which can be administered via systemic delivery (Figure 2A) to transduce neurons across the brain (28), we studied the $L a c Z$ signals (Figure 2B) and the protein expression level of Scn2a. We found that the FlpO treatment resulted in a partial but significant elevation of Nav1.2 protein expression in adult Scn2agtKo/gtKo mice compared to control PHP.eB.AAV transduction (Figure 2C). Remarkably, this partial restoration of Scn2a expression in adult mice translated into changes in neuronal excitability. We found that the adult Scn2agtKo/gtKo mice transduced with the AAV-FlpO displayed decreased neuronal excitability of striatal MSNs (Figure 2D-E). In the FlpO-treated group, the triggered AP firing of MSNs in Scn2agtKo/gtko mice was reduced to the WT range, together with the correction of other parameters including the RMP, AP waveform among others (Figure 2D-J). Collectively, our data show that even with a partial restoration of Scn2a expression to $~ 50-60 \%$ of WT expression level, we are still able to achieve an almost full rescue of neuronal excitability in adult mice.

In the cortico-striatal circuit, principal pyramidal neurons of the mPFC project to the striatum, and are suggested to be involved in seizure initiation. As the mPFC is also implicated in the absence-like seizures of $S c n 2 a^{+-}$mice $(16,17)$, we studied the excitability of layer $V$ pyramidal neurons of the mPFC. We found that the excitability of these neurons was increased significantly compared to the WT mice, and can be reversed by FlpO mediated partial restoration of Scn2a expression as well (Figure S2). Together, our data suggest that the Nav1.2 deficiency-related hyperexcitability exists along the cortico-striatal circuit, manifested in the principal neurons of both cortex and striatum brain regions.

The hyperexcitability seen in neurons with Nav1.2 deficiency could come from the altered intrinsic properties independent of other neurons (autonomous), or a result of a disrupted circuit. To distinguish these possibilities, we performed AAV injections of FlpOmCherry to transduce only a few MSNs in the CPu sparsely. We then performed patchclamp recordings on adjacent neurons with or without fluorescence (AAV-negative/nontransduced neurons versus AAV-positive/transduced neurons) (Figure 3A). Strikingly, our data showed that the transduced neurons (showing fluorescence) display greatly decreased neuronal excitability, compared to non-transduced neurons (showing nonfluorescence) in the same brain slices. In particular, we found that the RMP, input resistance, and the altered AP waveform were reversed in FlpO-transduced neurons of Scn2agtKo/gtko mice (Figure 3B-L). Moreover, when we performed the recordings at a fixed membrane potential of $-80 \mathrm{mV}$, similar findings could still be obtained (Figure S3AJ). On the other hand, non-transduced neurons displayed hyperexcitability similarly to neurons from Scn2agtKo/gtKo mice without virus transduction. Our data indicate that the hyperexcitability of each MSN can be modulated by the expression level of Scn2a autonomously, and the Nav1.2 deficiency-related hyperexcitability is the intrinsic property of a particular neuron independent of its surrounding neurons or circuit. 


\section{Downregulation of potassium channels contributes to the elevated action potential firings}

To reveal the possible molecular basis underlying the enhanced neuronal excitability of Scn2agtKO/gtKo mice, we studied the gene expression profile using RNA sequencing (RNAseq). We identified around nine hundred genes that were significantly up- or downregulated in Scn2agtKO/gtKo mice compared to WT littermates (Figure 4A). Scn2a expression was at $29.6 \%$ of the WT value (Figure 4B), consistent with our qPCR (Figure S4A) and Western blot study (Figure 2C and Figure S1C). Nav1.6 and Nav1.2 are two major sodium channels often working in a coordinated fashion in principal neurons in the CNS, and the dysfunction of Nav1.6 is involved in seizures (29-32). In Nav1.6-deficient mouse models, Nav1.2 was upregulated, suggesting a compensatory relationship (33, 34). Interestingly, we detected a slightly reduced expression of Nav1.6 in Scn2agtKO/gtKO mice in our RNA-seq analysis. This reduction of Nav1.6 did not reach statistical significance $(91.4 \pm 2.3 \%$ of $\mathrm{WT}, \mathrm{n}=4, \mathrm{p}=0.39)$ by $\mathrm{qPCR}$ validation (Figure S4A), indicating that our observed neuronal hyperexcitability is not likely to result from the compensation of the Nav1.6 channel expression.

Besides the Nav channels, potassium channels are also known to be major mediators setting the neuronal excitability $(35,36)$, and are often co-localized with Nav in the axon with high expression to regulate excitability $(37,38)$. Indeed, as the AP waveform was altered markedly in neurons with severe Nav1.2 deficiency, it is likely that the functions or expressions of potassium channels, which are responsible for many aspects of AP waveform, were disrupted in these neurons. Thus, we expanded our survey to include potassium channels. Notably, we found multiple potassium channel genes to be significantly downregulated (Kcne2, Kcng4, Kcnv1, Kcna1, Kcna2, Kcnj10, and Kcnk1) in our RNA-seq analysis (Figure 4B). As the top three genes are regulatory subunits or modifiers, we mainly focused on Kv1.1 and Kv1.2 (encoded by Kcna1 and Kcna2, respectively), which are known to be involved in seizures (39). Our qPCR experiment validated that Kv1.1 and Kv1.2 were significantly downregulated (Figure S4B).

To understand the contribution of potassium channels towards neuronal excitability, we tested pimaric acid (PiMA, $10 \mu \mathrm{M}$ ) on MSNs in the brain slices of Scn2agtKO/gtKo mice. PiMA is a relatively general $\mathrm{K}$ channel opener but with demonstrated properties as a Kv1.1-Kv2.1 opener (40). While it might not be surprising that PiMA can affect neurons from WT mice, it was quite remarkable that PiMA almost completely rescued the excitability of MSNs from Scn2agtKO/gtKo mice to the WT range (Figure 4CM). By pre-incubation of $10 \mu \mathrm{M}$ PiMA for $10 \mathrm{~min}$ or more, we found that PiMA could significantly rescue the AP firings of MSNs from Scn2agtKO/gtKo mice towards the WT range (Figure 4C-D). Strikingly, most of the parameters, including input resistance, RMP, AP rheobase, voltage threshold, amplitude, fAHP, and half-width values were reversed towards the WT values in the presence of PiMA as well (Figure 4E-M).

As a selective Kv1.1 opener (4-Trifluoromethyl-L-phenylglycine, 4TFMPG, 100 $\mu \mathrm{M}$ ) was recently reported (41), we further investigated the role of the specific Kv1.1 in MSNs of Scn2agtKo/gtKo mice. Notably, we found that the pre-incubation of $100 \mu \mathrm{M}$ 4TFMPG for 10 min or more could significantly reverse the hyperexcitability of MSNs as well as the RMP and rheobase values of Scn2agtKO/gtKo mice (Figure S4C-E, I, N-O, and T). Interestingly, different from the relatively broad potassium channels opener (PiMA), 
4TFMPG was not able to rescue the AP voltage threshold, amplitude, fAHP, half-width, or input-resistance (Figure S4F-M and Figure S4Q-X).

To further understand whether the change of expressions in these $\mathrm{Kv}$ channels is due to Nav1.2 deficiency, we performed the qPCR experiment with striatal tissues from mice injected with AAV-FIpO, in which the expression of Scn2a was restored. Our data revealed that after the restoration of Scn2a expression by FlpO, the expressions of Kv1.1 and Kv1.2 were increased (Figure S4B), suggesting that neurons have a dynamic adaptation mechanism to regulate gene expression in responses to the change of Scn2a expression level. Taken together, our data indicate that $\mathrm{K}_{v}$ channels (especially Kv1.1) are important mediators of the hyperexcitability phenotypes observed in MSNs with severe Nav1.2 deficiency.

\section{In vivo neuronal firing in the CPu region is enhanced in adult Nav1.2-deficient mice}

To test whether the enhanced neuronal excitability in brain slices translates into increased neuronal firing in vivo, we performed high-density Neuropixels recording on Scn2agtKo/gtKo and WT mice. The whole experiment pipeline consists of five steps, including surgery to implant headplate, recovery, recording, and postmortem imaging (Figure S5A). Mice were allowed to recover for 14 days before in vivo recording. The Neuropixels probe, consisting of $\sim 300$ recording electrodes across $3-\mathrm{mm}$ length, was inserted into the $\mathrm{CPu}$ region to record the neuronal firing of mice in their resting-state (Figure S5B-C). After spike-sorting, units were manually verified and clear action potential waveform could be identified from both Scn2agtKO/gtKo and WT mice (Figure S5D). A published set of criteria were used to isolate putative MSNs $(42,43)$. Notably, our data demonstrated that putative MSNs from the CPu region of Scn2agtKO/gtKo mice display a higher mean firing frequency compared to WT mice (Figure S5E). Together, our data suggest that the neuronal hyperexcitability observed in brain slice recording can manifest as enhanced in vivo firings of head-fixed mice in their resting-state.

\section{DISCUSSION}

Here in this paper, we report a counterintuitive finding that severe Nav1.2 deficiency renders hyperexcitability of principal MSNs in the striatum and pyramidal neurons in the mPFC, challenging the conventional paradigm. We further demonstrated that this hyperexcitability is reversible even in adult mice, showing a dynamic adaptive ability of neurons. Moreover, we provided evidence to suggest that the compensatory reduction in expressions of $\mathrm{Kv}$ channels is a possible mechanism underlying this hyperexcitability, revealing a remarkable interplay between neuronal excitability and gene regulation. In vivo study further demonstrated that this elevated neuronal excitability identified in brain slices can be translated into enhanced neuronal firing in live mice. Our data thus provided a plausible explanation for the mysterious epileptic seizure phenotypes in humans with SCN2A deficiency, and identified molecular targets for potential therapeutic interventions.

Nav1.2 channel plays a variety of roles in the initiation, propagation, and backpropagation of APs during development and adulthood $(20,44-47)$. In the early stage of development, Nav1.2 is suggested to be the main sodium channel expressed in the 
axon initial segment (AIS) $(1,48,49)$. Later in the development, Nav1.6 becomes the dominating channel in the axon and distal AIS, while the expression of Nav1.2 is redistributed to other parts of the neurons including proximal AIS and dendrites $(20,25,47$, 50). A recent study found that pyramidal neurons of the mPFC from adult Scn2a ${ }^{+/-}$mice have impaired excitatory postsynaptic current but intact AP firing (20). Here we revealed that severe Nav1.2 deficiency beyond a $50 \%$ reduction level in neurons surprisingly leads to hyperexcitability, which is an intrinsic property of the neurons that can be modulated in adulthood. However, how severe Nav1.2 deficiency changes neuronal excitability during early development remains to be determined. It is also worth noting that the expression of Nav1.2 in parvalbumin (PV) or somatostatin (SST) interneurons is limited $(5,20)$, and Nav1.2 does not seem to play a functional role in these interneurons (20). Nevertheless, it is still possible that while the expression of Nav1.2 in PV and SST interneurons is low, the severe reduction of Scn2a in principal neurons may result in compensatory adaptation, which indirectly affects the excitability of interneurons. It would be interesting to further explore these possibilities in a future study.

Because of the strong expression of SCN2A in principal neurons and its key roles to support AP firing, it is well accepted that increased Nav1.2 channel activity leads to enhanced excitability of principal neurons and aggravates seizures $(2,12,51)$. Intriguingly, Nav1.2 deficiency, which is mainly found in ASD/ID cases, and conventionally expected to impair neuronal excitability, is also associated with epilepsies $(6,10,52)$. It is estimated that $20 \sim 30 \%$ of ASD/ID patients with Nav1.2 deficiency develop "late-onset" seizures $(2,11)$. Treating epileptic seizures in these patients with Nav1.2 deficiency is extremely difficult, and the use of sodium channel blockers has been shown to exacerbate, rather than alleviate, the seizures (11). Our current data, together with published studies on Scn2a+/- mice (20), may suggest a new paradigm. A moderate deficiency of Nav1.2 (i.e., loss-of-function variants) may impair neuronal excitability contributing to ASD and ID, whereas a severe deficiency of Nav1.2 (i.e., protein-truncating variants) tips the balance, resulting in neuronal hyperexcitability and increased seizure susceptibility. Notably, an independent study by the Bender lab (Spratt et al, cosubmission) found that $100 \%$ knockout of Scn2a in a subset of pyramidal neurons in the $\mathrm{mPFC}$ results in hyperexcitability as well.

Potassium channels are known to play major roles in neuronal excitability and epileptic seizures (53-57). The AP waveform, which is highly influenced by the orchestration of a variety of potassium channels, is strongly disrupted in neurons with Nav1.2 deficiency, further suggesting the involvement of potassium channels. Our RNAseq results identified multiple potassium channels to be significantly downregulated in Scn2a ${ }^{\text {gtKO/gtKo }}$ mice, including voltage-gated potassium channels (i.e., Kv1.1 and Kv1.2), as well as two-pore potassium channels (Kcnk1) and inward rectifier potassium channels (Kcnj10) (Figure 4A-B). Kv1.1, for example, is abundantly expressed in principal neurons of the CNS, and contributes to the threshold as well as the interspike intervals during repetitive firing (35). Additionally, it is known that Kv1.1 can form heteromultimeric channels with Kv1.2 (35), which was identified to be downregulated in Scn2agtKO/gtKo mice by our RNA-seq analysis as well.

In this current study, we have obtained evidence to show that PiMA markedly reverses the elevated action potential firings associated with severe Nav1.2 deficiency 
(Figure 4C-D). PiMA is a relatively general potassium channels opener, with demonstrated properties as a Kv1.1-Kv2.1 and large-conductance $\mathrm{Ca}^{2+}$-activated $\mathrm{K}$ channel (BK) opener (40). It is worth noting that besides increased action potential firings, neurons from Scn2agtKO/gtKo mice display reduced action potential amplitude, higher voltage-threshold, increased input resistance, and elevated fAHP (Figure 1 and Figure S1), which could be modulated by different potassium channels $(58,59)$. The reduced driving force on $\mathrm{Kv}_{v}$ channels due to the change of AP waveform, including $\mathrm{AAH}$, might contribute to the hyperexcitability phenotypes as well. Nevertheless, PiMA turns out to rescue most of these altered parameters (Figure 4), which is unexpected but may indicate that PiMA has additional targets beyond $\mathrm{Kv}_{\mathrm{v}} 1.1-\mathrm{Kv} 2.1$ and $\mathrm{BK}$ channels. Importantly, a relatively selective $\mathrm{Kv1.1}$ opener (4TFMPG) is also able to reduce neuronal hyperexcitability, suggesting that the enhanced action potential firing in Scn2agtKo/gtKo mice could be largely attributed to the Kv1.1 channel (Figure S4C-E, N-O). Notably, 4TFMPG was not able to restore the input resistance, AP voltage threshold, amplitude, fAHP, or half-width values (Figure S4F-M and Figure S4Q-X), largely fitting the notion that 4TFMPG is selective for Kv1.1 (41). Viral delivery of a specific gene is a different approach to elucidate the precise role of each distinct potassium channel towards neuronal excitability. Indeed, AAV-Kv1.1 has been suggested as novel gene therapy to reduce seizures $(60,61)$. It would be appealing to test the effect of AAV-Kv1.1 in Scn2agtKO/gtKo mice in a follow-up study. However, as several other potassium channels were also found to be downregulated, a multiple-gene delivery approach might be needed to deeply assess the contributions of this collection of ion channels toward neuronal hyperexcitability.

In summary, our results reveal an unexpected hyperexcitability phenotype in neurons with severe Nav1.2 deficiency, which is reversible and likely due to the compensatory reduction in expressions of potassium channels. The maladaptation or "over-compensatory" from potassium channels is likely a cause leading to the hyperexcitability of neurons to promote seizures. While it is a demonstrated clinical observation that patients with SCN2A deficiency often develop intractable seizures, there are no disease models that exist thus far for mechanistic investigation of this observation. Neuronal hyperexcitability identified in this unique Nav1.2-deficient mouse model is the first step towards the understanding of disease mechanisms underlying severe SCN2A deficiency. Our findings may explain the puzzling clinical observation that a portion of patients with Nav1.2 deficiency still develop seizures, and guide further development of interventions targeting $\mathrm{K} v$ channels to treat Nav1.2 deficiency-related disorders (62).

\section{MATERIALS AND METHODS}

\section{Mouse strains}

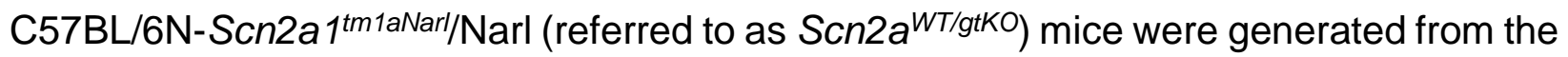
National Laboratory Animal Center Rodent Model Resource Center based on a modified gene-trap design $(23,24)$. The generation and basic characterization of this mouse model are available in our recent article (22). The targeting construct (tm1 a trapping cassette) was electroporated into $\mathrm{C} 57 \mathrm{BL} / 6 \mathrm{~N}$ embryonic stem cells, and founders in a pure 
experiments were approved by the Institutional Animal Care and Use Committee (IACUC). Mice were same-sex housed in mixed-genotype groups (3-5 mice per cage) on vented cage racks with 1/8" Bed-o-cobb bedding (Anderson, Maumee, OH, USA) and > $8 \mathrm{~g}$ of nesting material as enrichment (shredded paper, crinkle-cut paper, and/or cotton nestles) on a 12hr light cycle. Food (2018S Teklad from Envigo) and reverse osmosis water were given ad-lib. Heterozygous (HET, Scn2aWT/gtKO) mice were used as breeding pairs to obtain homozygous (HOM, Scn2agtKO/gtKO) mice and WT littermates for study. Whenever possible, investigators were blind to the genotype of the mice.

\section{Reagents}

Reagents used were as follows: $\mathrm{N}$-(2-aminoethyl) biotin amide hydrochloride (NEUROBIOTIN ${ }^{\text {TM }}$ Tracer, SP-1120, from Vector Laboratories), Alexa 488-conjugated streptavidin (Molecular Probes, Eugene, OR, USA), Tetrodotoxin citrate (sodium channel blocker) was solubilized in pure water at a stock concentration of $500 \mu \mathrm{M}$ ); Pimaric acid (PiMA, Kv channels opener) was solubilized in DMSO at a $1000 \times$ stock concentration of $10 \mathrm{mM}$; 4-(Trifluoromethyl)-L-phenylglycine (4TFMPG, Kv1.1 specific opener) was solubilized in $1 \mathrm{M}$ hydrochloric acid at a $1000 \times$ stock concentration of $100 \mathrm{mM}$; (2Fluorophenyl) glycine (2FPG, Kv1.1 specific opener) was solubilized in $0.25 \mathrm{M}$ hydrochloric acid at a $1000 \times$ stock concentration of $100 \mathrm{mM}$.

\section{Antibodies}

Primary antibodies used were: Rabbit anti-SCN2A (Nav1.2) (1: 1000, Alomone Labs, ASC-002), mouse anti- $\beta$-Actin (1:2000, Cell Signaling Technology, 3700S), and GAPDH (D16H11) XP ${ }^{\circledR}$ Rabbit mAb (1:2000, Cell Signaling Technology, 5174S). Secondary antibodies were: IRDye® 680RD Goat anti-Rabbit IgG Secondary Antibody (1:5000, LICOR Biosciences, AB_10956166) and IRDyeß 680RD Goat anti-Mouse IgG Secondary Antibody (1:5000, LI-COR Biosciences, AB_10956588).

\section{Genotyping}

Mice were labeled and genotyped via ear punch at weaning (21-28 days old). Genotyping for the tm1a cassette was performed using gene-specific polymerase chain reaction (PCR) on DNA extracted from ear tissues with a tissue DNA extraction kit (MachereyNagel, Bethlehem, PA, USA) with primers (forward 5' to 3': GAGGCAAAGAATCTGTACTGTGGGG, reverse: 401 GACGCCTGTGAATAAAACCAAGGAA). The wild type allele's PCR product is 240 base pairs (bp) and the tm1a ( $g$ tKO) allele's PCR product is $340 \mathrm{bp}$.

\section{Adeno-associated virus (AAV) production}

pAAV-EF1a-mCherry-IRES-Flpo was a gift from Karl Deisseroth (63) (Addgene plasmid \# 55634 and viral prep \# 55634-AAVrg; http://n2t.net/addgene:55634; RRID: Addgene_55634), AAV9-PHP.eB-EF1a-mCherry-IRES-Flpo with the titer of $2.56 \times 10^{13}$ 
$\mathrm{GC} / \mathrm{mL}$ was packed by Penn Vector Core (http://pennvectorcore.med.upenn.edu/);

409 Control virus, PHP.eB-Ef1a-DO-mCherry-WPRE-pA with the titer of $1.2 \times 10^{13} \mathrm{GC} / \mathrm{mL}$ was

410 packed by Bio-Detail Corporation.

\section{Surgical procedures}

413 For all surgeries (except as noted), mice were systemically anesthetized with ketamine and xylazine, and received analgesic buprenorphine to help postoperative recovery.

\section{AAV injections}

For systemic delivery of virus, each adult mouse received $2 \times 10^{11}$ infections of FlpO- or control AAV virus via tail vein injection. For viral injection into the brain to label neurons sparsely, mice were anesthetized with ketamine/xylazine (100/10 mg/kg, i.p.) and secured in a stereotaxic apparatus with ear-bars (RWD Ltd, China). After exposing the skull via a small incision, small holes for each hemisphere were drilled for injection based on coordinates to bregma. Mice were bilaterally injected with AAV virus (diluted into $\sim 5 \times 10^{10}$ infections units per $\mathrm{mL}$ with PBS) into the caudate nucleus and the putamen (CPu, dorsal striatum) (coordinates of the injection sites relative to bregma: $A P+1.30 \mathrm{~mm}$, $\mathrm{ML} \pm 1.25 \mathrm{~mm}$, DV $-3.30 \mathrm{~mm}$; AP $+0.50 \mathrm{~mm}, \mathrm{ML} \pm 2.00 \mathrm{~mm}$, DV -3.25 mm, 0.5-1 $\mu \mathrm{L}$ per point) and the nucleus accumbens (NAc, ventral striatum) (coordinates of the injection sites relative to bregma: $\mathrm{AP}+1.30 \mathrm{~mm}, \mathrm{ML} \pm 1.25 \mathrm{~mm}, \mathrm{DV}-4.50 \mathrm{~mm}, 0.5-1 \mu \mathrm{L}$ per point) with sharpened glass pipettes (Sutter Instrument), self-made to have a bevel of $35^{\circ}$ and an opening of $20-\mu \mathrm{m}$ diameter at the tip (64), attached to syringe needles $(200-\mu \mathrm{m}$ diameter). The pipette was filled from the back end with mineral oil and attached to a syringe needle mounted in a microinjection syringe pump (World Precision Instruments, UMP3T-2). Before injection, the viral suspension was suctioned through the tip of the pipette. The skull over the target coordinates was thinned with a drill and punctured with the tip of the pipette. The pipette was inserted slowly $(120 \mu \mathrm{m} / \mathrm{min})$ to the desired depth. The virus was slowly ( 100-150 nL/min) injected to the desired location. Before being retracted out of the brain, the pipette was left at the same place for 10 min when the injection was finished. The virus was allowed to express for at least three weeks before electrophysiological recordings. Animals were allowed to recover from surgery for one week and their body weight and health conditions were closely monitored during recovery. The accurate location of injection sites and viral infectivity were confirmed in all mice posthoc by imaging of sections ( $50 \mu \mathrm{m}$ in thickness) containing the relevant brain regions.

\section{Perfusions and tissue processing}

444 For immunostaining, mice were administered an overdose of anesthesia and transcardiacally perfused with ice-cold PBS followed by $4 \%$ paraformaldehyde (PFA) (For LacZ staining, 4\% PFA was replaced by $2 \%$ formaldehyde $+0.2 \%$ glutaraldehyde in PBS, hereinafter inclusive). After perfusion, brain slices were dissected out and post-fixed in $4 \%$ PFA overnight at $4^{\circ} \mathrm{C}$. Tissues were cryoprotected by sinking in gradient sucrose $449(10 \%, 20 \%$, and $30 \%)$ with $0.01 \mathrm{M}$ PBS at $4^{\circ} \mathrm{C}$ and subsequently frozen in $20 \%$ sucrose 450 and $30 \%$ sucrose in $1 \times$ phosphate-buffered saline (PBS) for 24-48 hrs. Samples were 
frozen in Optimal Cutting Temperature compound using dry ice and stored at $-80^{\circ} \mathrm{C}$. Tissue sections of $20 \mu \mathrm{m}$ in thickness were taken on a cryostat (Leica CM1950) and allowed to air dry on slides, followed by analysis on a confocal microscope (Zeiss LSM 900 or Nikon A1R-MP).

\section{LacZ ( $\beta$-galactosidase) staining}

Both Scn2agtKO/gtKo and WT mice with or without AAV injection were processed at the same time under the same condition to minimize variation. Cryosections were fixed with $2 \%$ formaldehyde $+0.2 \%$ glutaraldehyde in PBS for 5 min. Then sections were washed at least 5 min in PBS (with $0.02 \%$ Triton X-100 for optimal reduction of unspecific binding of antibodies). Tissues were covered with a volume of freshly prepared staining solution [X-Gal solution added into Iron Buffer (1/19, v/v) and mixed thoroughly for $10 \mathrm{~min}$ ], sufficient to fully cover the specimen (e.g., $50 \mu \mathrm{L}$ ) and incubate for $15-30 \mathrm{~min}$ at $37^{\circ} \mathrm{C}$ in a humid chamber until cells were stained blue. Color development was checked under a microscope and incubation time was continued if necessary. Specimen were washed three times with PBS and mounted in glycerol before storage after removing PBS. Images were analyzed under an upright light microscope.

\section{Immunostaining and imaging analysis}

Cryosections (20 $\mu \mathrm{m}$ in thickness) were permeabilized, incubated in blocking buffer $(0.5 \%$ Triton X-100 and 5\% normal goat serum in PBS) for one hour at room temperature, and overlaid with primary antibodies overnight at $4^{\circ} \mathrm{C}$. Then, the corresponding Alexa Fluor 488-, 594- or 647-conjugated secondary antibodies were applied. All stained sections were mounted with DAPI-containing mounting solution and sealed with glass coverslips. All immunofluorescence-labeled images were acquired using a confocal microscope (65).

\section{RNA sequencing}

RNA extraction: Four Scn2agtKo/gtKo (HOM) and four WT littermate mice were used to extract RNA. Mice were given an overdose of anesthesia and transcardiacally perfused with ice-cold PBS. Acute coronal brain slices containing cortex and striatum (300- $\mu \mathrm{m}$ in thickness) were cut using a vibratome (Leica VT1200S, Germany). Cortex and striatum were rapidly microdissected, immersed into liquid nitrogen, and stored at $80^{\circ} \mathrm{C}$ until use (same procedures for Western Blotting and qPCR). Based on the manufacturer's instructions, total RNAs were extracted with TRIzol reagent (Thermo Fisher Scientific, 15596018) from mouse cerebral tissues.

Library preparation and sequencing: Novogene prepared libraries using the TruSeq Stranded kit (Illumina, San Diego, CA) and RNA quality was assessed using an Agilent Nano RNA ChIP. Paired-end 150 bp reads were sequenced using the NovaSeq 6000.

Analysis: Reads were quality trimmed and Illumina TruSeq adapter sequences were removed using Trimmomatic v.0.36 (66). A sliding window approach to trimming was performed, using a window size of 5 and a required average Phred (quality) score of 
16. Bases falling below a Phred score of 10 at the start and end of reads were trimmed and reads shorter than 20 bases in length after trimming were removed. FastQC v. 0.11.7 (67) was run to observe data quality before and after trimming/adapter removal. STAR v. 2.5.4b (68) was used to align reads to the Ensembl Mus musculus genome database version GRCm38.p6. The htseq-count script in HTSeq v.0.7.0 (69) was run to count the number of reads mapping to each gene. HTSeq used Biopython v.2.7.3 in the analysis. HTSeq was run utilizing the GTF file on "intersection-nonempty" mode. The HTSeq feature was set to "exon" and the attribute parameter was set to "gene id" and the -stranded=reverse option was set. The Bioconductor packages DESeq $\overline{2}$ v.1.22.2 and edgeR 3.24.3 were used for differential expression analysis. Genes that were identified as differentially expressed in both packages were used as high confidence differentially expressed genes and were used in subsequent pathway analysis. The BenjaminiHochberg false discovery rate correction was used to correct p-values for multiple testing. To improve power, low expression transcripts were filtered out of the data before performing differential expression analysis. The threshold chosen was to filter out all genes expressed at lower than 0.5 counts per million (CPM) in all samples combined. After filtering, 18,134 genes were remaining. The expression of genes between WT and $\mathrm{HOM}$ were deemed significant if the adjusted $\mathrm{p}$-value $<0.05$. The Bioconductor package biomaRt v. 2.38.0 was used to perform annotation of genes. ClusterProfiler v. 3.10.1 was used to perform pathway and gene ontology enrichment analysis.

\section{Western blotting}

Brain tissues were homogenized in ice-cold RIPA lysis and extraction buffer (Thermo Fisher Scientific, 89901) supplemented with protease and phosphatase inhibitors (Thermo Fisher Scientific, A32953), sonicated, and cleared by centrifugation (10,000× $\mathrm{g}$, $10 \mathrm{~min}$, at $\left.4^{\circ} \mathrm{C}\right)$. Protein concentration in the supernatant was determined by (determined by Nanodrop, Thermo Scientific). Proteins in 1x sample buffer [62.5 mM Tris-HCl $(\mathrm{pH}$ 6.8 ), $2 \%(\mathrm{w} / \mathrm{v})$ SDS, $5 \%$ glycerol, $0.05 \%(\mathrm{w} / \mathrm{v})$ bromophenol blue] were denatured by boiling at $95^{\circ} \mathrm{C}$ for $5 \mathrm{~min}$. For each sample, $40 \mu \mathrm{g}$ total proteins were loaded to the $8 \%$ sodium dodecyl sulfate-polyacrylamide (SDS-PAGE) gels and transferred onto PVDF membrane (Millipore, IPFL00010) by electrophoresis. Blots were blocked in 5\% nonfat milk in Tris-buffered saline and Tween 20 (TBST) for $1 \mathrm{~h}$ at room temperature and probed with the primary antibody in $5 \%$ milk-TSBT overnight at $4^{\circ} \mathrm{C}$. After overnight incubation, the blots were washed three times in TBST for $15 \mathrm{~min}$, followed by incubation with corresponding IRDye ${ }^{\circledR}$ 680RD secondary antibodies in TBST for $2 \mathrm{~h}$ at room temperature. Following three cycles of 15 min washes with TBST, the immunoreactive bands were scanned and captured by the Odyssey ${ }^{\circledR}$ CLx Imaging System (LI-COR Biosciences) and quantitatively analyzed by densitometry with Image Studio Lite 5.2 (LI-COR Biosciences) or ImageJ software (NIH). Each sample was normalized to its $\beta$-actin or GAPDH, then normalized with the corresponding WT littermates.

\section{RNA isolation, reverse transcription, and qPCR analysis}

Total RNAs were extracted with TRIzol reagent (Thermo Fisher Scientific, 15596018) from mouse cerebral tissues according to the manufacturer's instructions. $4 \mu \mathrm{g}$ RNA was 
subjected to reverse transcription (RT) with a Maxima First Strand cDNA Synthesis Kit (Thermo Fisher Scientific, K1672). The resulting cDNAs were subjected to quantitative PCR analysis using the PowerUp ${ }^{\text {TM }}$ SYBR ${ }^{\text {TM }}$ Green Master Mix (Thermo Fisher Scientific, A25777) and specific primers in a C1000 Touch PCR thermal cycler (Bio-Rad). Gapdh and $\beta$-actin mRNA levels were used as an endogenous control for normalization using the $\Delta \mathrm{Ct}$ method (70). In brief, test $(\mathrm{T}): \Delta \mathrm{Ct}^{\top}=\left[\mathrm{Ct}^{\top}\right.$ (target gene) - $\mathrm{Ct}^{\top}$ (internal control)]; Amount of the target $=2^{-\Delta \mathrm{Ct}}$.

\section{Patch-clamp recordings}

Acute slice preparations: Electrophysiology was performed in slices prepared from 2-5 months-old Scn2agtKO/gtKo and corresponding control mice. Mice were deeply anesthetized with ketamine/xylazine (100/10 mg/kg, i.p., $0.1 \mathrm{~mL}$ per 10 grams of body weight), and then transcardially perfused, and decapitated to dissect brains into ice-cold slicing solution containing the following (in $\mathrm{mM}$ ): 110 choline chloride, $2.5 \mathrm{KCl}, 1.25$ $\mathrm{NaH}_{2} \mathrm{PO}_{4}, 25 \mathrm{NaHCO}_{3}, 0.5 \mathrm{CaCl}_{2}, 7 \mathrm{MgCl}_{2}, 25$ glucose, 0.6 sodium ascorbate, 3.1 sodium pyruvate (bubbled with $95 \% \mathrm{O}_{2}$ and $5 \% \mathrm{CO}_{2}, \mathrm{pH} 7.4,305-315 \mathrm{mOsm}$ ). Acute coronal slices containing PFC and/or striatum (300- $\mu \mathrm{m}$ in thickness) were cut by using a vibratome (Leica VT1200S, Germany) and transferred to normal artificial cerebrospinal fluid (aCSF) (in mM): $125 \mathrm{NaCl}, 2.5 \mathrm{KCl}, 2.0 \mathrm{CaCl}_{2}, 2.0 \mathrm{MgCl}_{2}, 25 \mathrm{NaHCO}_{3}, 1.25$ $\mathrm{NaH}_{2} \mathrm{PO}_{4}, 10$ glucose (bubbled with $95 \% \mathrm{O}_{2}$ and $5 \% \mathrm{CO}_{2}, \mathrm{pH} 7.4,305-315 \mathrm{mOsm}$ ). Then, slices were incubated at $37^{\circ} \mathrm{C}$ for $20-30$ minutes and stored at room temperature before use. Slices were visualized under IR-DIC (infrared-differential interference contrast) using a BX-51WI microscope (Olympus) with an IR-2000 camera (Dage-MTI).

Ex vivo electrophysiological whole-cell recordings: All somatic whole-cell patch-clamp recordings were performed from identified striatal MSNs or mPFC layer $\mathrm{V}$ pyramidal neurons. The selection criteria for MSNs were based on morphological characteristics with medium-sized cell body presenting polygon or diamond viewed with a microscope equipped with IR-DIC optics (BX-51WI, Olympus), and numerous dendritic spines and their hyperpolarized RMP (lower than $-80 \mathrm{mV}$ ) based on published method (71). Layer $\mathrm{V}$ pyramidal cells with a prominent apical dendrite were visually identified mainly by location, shape, and pClampex online membrane test parameters. Putative pyramidal cells in layer $5 b$ were identified based on regular spiking characteristics (20, $72,73)$. To minimize variability, recordings were made on cells with low or high HCN expression levels, corresponding to intratelencephalic (IT) or pyramidal tract (PT) neurons, respectively. The selection criterion for $\mathrm{PT}$ pyramidal cells was based on their firing properties and shape of the AP (i.e., all cells' intrinsic ability to generate, upon subthreshold depolarization possessed a prominent after-hyperpolarization and significant membrane-potential sags induced by both hyperpolarizing and depolarizing current injection at the soma). Recordings of PT neurons were used for further analysis.

For whole-cell current-clamp recordings, the internal solution contained (in $\mathrm{mM}$ ): $122 \mathrm{KMeSO}_{4}, 4 \mathrm{KCl}, 2 \mathrm{MgCl}_{2}, 0.2$ EGTA, $10 \mathrm{HEPES}, 4 \mathrm{Na} 2 \mathrm{ATP}, 0.3$ Tris-GTP, 14 Trisphosphocreatine, adjusted to $\mathrm{pH} 7.25$ with $\mathrm{KOH}, 295-305 \mathrm{mOsm}$. The sag ratio, input resistance, and firing number were obtained in response to a series of $400 \mathrm{~ms}$ current steps from $-200 \mathrm{pA}$ to $+400 \mathrm{pA}$ in increments of $50 \mathrm{pA}$, each sweep duration of $5 \mathrm{~s}$ with 
cells held at the normal RMP or a fixed potential of $-80 \mathrm{mV}$. The sag ratio was calculated with the equation:

$$
\text { Sag ratio }=\left(\mathrm{V}_{\text {baseline }}-\mathrm{V}_{\text {steady-state }}\right) /\left(\mathrm{V}_{\text {baseline }}-\mathrm{V}_{\text {min }}\right)
$$

Where $V_{\text {baseline }}$ is the resting membrane potential or $-80 \mathrm{mV}, V_{\min }$ is the minimum voltage reached soon after the hyperpolarizing current pulse, and $V_{\text {steady-state }}\left(V_{\text {ss }}\right)$ is the voltage recorded at 0-10 ms before the end of the $-200 \mathrm{pA}$ stimulus.

The input resistance was calculated with the equation:

$$
\text { Input resistance }=\left(\mathrm{V}_{\text {baseline }}-\mathrm{V}_{\text {steady-state }}\right) * 10(\mathrm{M} \Omega)
$$

Where $V_{\text {baseline }}$ is the resting membrane potential or $-80 \mathrm{mV}$, and $V_{\text {steady-state }}\left(V_{s s}\right)$ is the voltage recorded at $0-10 \mathrm{~ms}$ before the end of the $-100 \mathrm{pA}$ stimulus.

The RMP, AP threshold, amplitude, fast afterhyperpolarization (fAHP), and halfwidth values were obtained in response to a $20 \mathrm{~ms}$ current step of the smallest current to obtain an intact AP, each sweep duration of $1.5 \mathrm{~s}$ and start-to-start intervals of $10 \mathrm{~s}$ with cells held at the normal RMP or a fixed potential of $-80 \mathrm{mV}$. The RMP, AP threshold, amplitude, fAHP, and half-width values were analyzed using the Clampfit 11.1 inbuilt statistics measurements program (Criteria as the baseline, peak amplitude, antipeak amplitude, and half-width). The threshold was defined as the $\mathrm{Vm}$ when $\mathrm{dV} / \mathrm{dt}$ measurements first exceeded $15 \mathrm{~V} / \mathrm{s}$.

We used thin-wall borosilicate pipettes (BF150-110-10) with open-tip resistances of 3-5 M $\Omega$. All recordings were started at least 1 min after breakin to stabilize the contact between the glass electrode and the cell membrane, and finished within $10 \mathrm{~min}$ to avoid large voltage changes due to the internal solution exchange equilibrium. Recordings were performed with an Axon MultiClamp 700B amplifier (Molecular Devices) and data were acquired using pClamp 11.1 software at the normal RMP or a fixed potential of $-80 \mathrm{mV}$, filtered at $2 \mathrm{kHz}$ and sampling rate at $20 \mathrm{kHz}$ with an Axon Digidata 1550B plus HumSilencer digitizer (Molecular Devices). Slices were maintained under continuous perfusion of aCSF at $32-33^{\circ} \mathrm{C}$ with a $2-3 \mathrm{~mL} / \mathrm{min}$ flow. In the whole-cell configuration series resistance (Rs) 15-30 M , and recordings with unstable Rs or a change of Rs > $20 \%$ were aborted.

To study the effect of Kv channels openers, the $1000 \times$ stocks were freshly diluted with aCSF, respectively. After 10 min perfusion of each opener or the corresponding vehicle control (0.1\% DMSO in aCSF for PiMA, and aCSF for 4TFMPG), the target neurons were studied with the continuous perfusion of the chemicals. One or two neurons were patched for each brain slice, and recordings were discarded if a slice was perfused with $\mathrm{Kv}$ channels openers for more than $30 \mathrm{~min}$.

For cell labeling, the internal solution contains $0.1-0.2 \%(\mathrm{w} / \mathrm{v})$ neurobiotin tracer. At the end of the electrophysiological recording (about $30 \mathrm{~min}$ ), slices were treated as previously described (74). Briefly, sections were fixed in $4 \%$ paraformaldehyde in $0.1 \mathrm{M}$ phosphate buffer $(\mathrm{pH} 7.4)$ for 20-30 min at room temperature, and subsequently washed 3-4 times for $30 \mathrm{~min}$ in $0.1 \mathrm{M}$ phosphate-buffered saline (PBS, pH 7.4) at $4^{\circ} \mathrm{C}$. Sections were then incubated in Alexa 488-conjugated streptavidin (overnight at $4^{\circ} \mathrm{C}, 1: 250$ in blocking solution) to visualize neurobiotin. 


\section{Neuropixels recordings and data analysis}

625

626

627

628

629

630

631

632

633

634

635

636

637

638

639

640

641

642

643

644

645

646

647

648

649

650

651

652

653

654

655

656

657

658

659

660

661

662

663

664

665

666

Surgeries: Animal preparation was performed as described previously $(64,75)$. Mice were anesthetized and head-fixed and underwent stereotaxic surgery to implant a metal headframe with a 10-mm circular opening (Narishige, MAG-1, and CP2) for headfixation. An incision was made over the skin. The skin and periosteum were removed, and a thin layer of cyanoacrylate (Krazy glue) was applied to attach the headplate and cover the exposed skull. A layer of clear Stoelting ${ }^{\text {TM }}$ Dental Cement (Fisher Scientific, 10000-786) was then applied on top of cyanoacrylate and forms a chamber around the skull to contain the ground wire and aCSF during electrophysiological recordings. The animals received two weeks recovery period after surgery before commencing experiments. Before electrophysiological recordings, a $600 \mu \mathrm{m}$ diameter craniotomy was prepared to access the intended brain regions with Neuropixels probes.

In vivo recordings: Electrophysiological recordings were made with Neuropixels probes in head-fixed mice. On the day of the experiment, the mouse was placed under light isoflurane anesthesia. A ground wire was secured to the skull, and the exposed brain was covered with a layer of $4 \%$ agar in aCSF. Following recovery from anesthesia, the mouse was head-fixed on the experimental rig. Before insertion, the probe tip was painted with CM-Dil. Briefly, the Neuropixels probe was secured to an arm of stereotaxic, and the backside of the probe was dipped into a $1 \mu \mathrm{L}$ droplet of CM-Dil (Thermo Fisher Scientific, C7000) dissolved in ethanol $(1 \mu \mathrm{g} / \mu \mathrm{L})$. The ethanol was allowed to evaporate, and the CM-Dil was dried onto the backside of the tip. The probe was then inserted slowly (120$480 \mu \mathrm{m} / \mathrm{min}$ ) into the striatum (coordinates of the injection sites relative to bregma: AP $+1.30 \mathrm{~mm}, \mathrm{ML} \pm 1.25 \mathrm{~mm}, 4.50 \mathrm{~mm}$ in depth) through the craniotomy on the skull. After reaching the desired depth for a probe, the probe was allowed to settle for 10 minutes before the commencement of recording. The first 384 electrodes were turned on in the Neuropixel probe, which corresponds to about $3.8 \mathrm{~mm}$ length probe. At the end of each recording session, the probe was retracted out of the brain and cleaned using Tergazyme (Alconox) followed by washing with distilled water. The probe insertion was verified by identifying the Dil fluorescence in sectioned brain tissue.

Data acquisition and Analysis: All data were acquired with a $30-\mathrm{kHz}$ sampling rate under the Open Ephys GUI (https://openephys.atlassian.net/wiki/spaces/OEW/pages/963280903/Neuropix-PXI). A 300-Hz highpass filter was present in the Neuropixels probe, and another $300-\mathrm{Hz}$ high-pass filter (3rdorder Butterworth) was applied offline before spike sorting.

Spike waveforms were automatically extracted from the raw data using Kilosort 2.0 (https://github.com/MouseLand/Kilosort/releases/tag/v2.0). The outputs were loaded into $\mathrm{PHY}$ (76) for manual refinement, which consisted of merging and splitting clusters, as well as marking non-neural clusters as "noise". Noise units were identified by their abnormal waveform shape, as well as distinct cyclical patterns in the autocorrelogram. A set of heuristic rules based on the features of waveforms to remove abnormal waveforms [the parameters were used for this purpose were peak-to-trough (PT) ratio $<0.99$ and recovery slope $<0]$. Waveforms for each unit were extracted from the raw data, and then averaged. All the averaged waveforms were used to calculate the mean waveform. 
Striatal single units were classified according to the methods described previously (42), using mean firing rate, mean waveform peak width at half-maximum, mean waveform trough width at half-minimum, and ISI distribution. These values were averaged across epochs when a cell was present in multiple epochs. The standard classification for the clusters was defined as follows: fast-spiking interneurons (FSIs): firing rate $>3 \mathrm{~Hz}$, peak width $<0.2 \mathrm{~ms}$, and a ratio of trough width to peak width (TPR) $<2.7$ (TPR was estimated by k-means clustering and was more reliable than exact trough width for FSIs); tonically-active neurons (TANs): $<5 \%$ of ISIs less than $10 \mathrm{~ms}$, a median ISI > $100 \mathrm{~ms}$, and peak width (0.2-0.35 ms) and trough width $(0.1-0.2 \mathrm{~ms})$ above the $95^{\text {th }}$ percentile for the remainder of the units; unclassified units had low TPR and/or narrow trough widths (< $0.3 \mathrm{~ms}$ ) but firing rates $<2 \mathrm{~Hz}$; all other units were considered putative medium spiny neurons (MSNs).

\section{Quantification and statistical analysis}

Normality and variance similarity was measured by GraphPad Prism before we applied any parametric tests. Two-tailed Student's $t$-test (parametric) or unpaired two-tailed Mann-Whitney U-test (non-parametric) was used for single comparisons between two groups. Other data were analyzed using one-way or two-way ANOVA with Tukey correction (parametric) or Kruskal-Wallis with Dunn's multi comparison correction (nonparametric) depending on the appropriate design. Post hoc comparisons were carried out only when the primary measure showed statistical significance. Error bars in all figures represent mean \pm SEM. $p$ values less than 0.05 were considered statistically significant. Statistical significance of differences at $p<0.05$ is indicated as one asterisk $\left({ }^{*}\right), p<0.01$ is indicated as two asterisks $\left({ }^{* *}\right)$, and $p<0.001$ is indicated as three asterisks $\left({ }^{\star \star *}\right)$ in all figures. Mice with different litters, body weights, and sexes were randomized and assigned to different treatment groups, and no other specific randomization was used for the animal studies.

\section{SUPPLEMENTARY MATERIALS}

Supplementary material for this article is available online at TBD.

\section{REFERENCES AND NOTES}

1. E. V. Gazina, B. T. Leaw, K. L. Richards, V. C. Wimmer, T. H. Kim, T. D. Aumann, T. J. Featherby, L. Churilov, V. E. Hammond, C. A. Reid, S. Petrou, 'Neonatal' Nav1.2 reduces neuronal excitability and affects seizure susceptibility and behaviour. Hum Mol Genet 24, 1457-1468 (2015).

2. S. J. Sanders, A. J. Campbell, J. R. Cottrell, R. S. Moller, F. F. Wagner, A. L. Auldridge, R. A. Bernier, W. A. Catterall, W. K. Chung, J. R. Empfield, A. L. George, Jr., J. F. Hipp, O. Khwaja, E. Kiskinis, D. Lal, D. Malhotra, J. J. Millichap, T. S. Otis, S. Petrou, G. Pitt, L. F. Schust, C. M. Taylor, J. Tjernagel, J. E. Spiro, K. J. Bender, Progress in Understanding and Treating SCN2A-Mediated Disorders. Trends in neurosciences 41, 442-456 (2018).

3. C. Tian, K. Wang, W. Ke, H. Guo, Y. Shu, Molecular identity of axonal sodium channels in human cortical pyramidal cells. Front Cell Neurosci 8, 297 (2014). 
4. H. Miyazaki, F. Oyama, R. Inoue, T. Aosaki, T. Abe, H. Kiyonari, Y. Kino, M. Kurosawa, J. Shimizu, I. Ogiwara, K. Yamakawa, Y. Koshimizu, F. Fujiyama, T. Kaneko, H. Shimizu, K. Nagatomo, K. Yamada, T. Shimogori, N. Hattori, M. Miura, N. Nukina, Singular localization of sodium channel beta4 subunit in unmyelinated fibres and its role in the striatum. Nature communications 5, 5525 (2014).

5. T. Yamagata, I. Ogiwara, E. Mazaki, Y. Yanagawa, K. Yamakawa, Nav1.2 is expressed in caudal ganglionic eminence-derived disinhibitory interneurons: Mutually exclusive distributions of Nav1.1 and Nav1.2. Biochemical and biophysical research communications 491, 1070-1076 (2017).

6. S. J. Sanders, M. T. Murtha, A. R. Gupta, J. D. Murdoch, M. J. Raubeson, A. J. Willsey, A. G. Ercan-Sencicek, N. M. DiLullo, N. N. Parikshak, J. L. Stein, M. F. Walker, G. T. Ober, N. A. Teran, Y. Song, P. El-Fishawy, R. C. Murtha, M. Choi, J. D. Overton, R. D. Bjornson, N. J. Carriero, K. A. Meyer, K. Bilguvar, S. M. Mane, N. Sestan, R. P. Lifton, M. Gunel, K. Roeder, D. H. Geschwind, B. Devlin, M. W. State, De novo mutations revealed by whole-exome sequencing are strongly associated with autism. Nature 485, 237-241 (2012).

7. A. Hoischen, N. Krumm, E. E. Eichler, Prioritization of neurodevelopmental disease genes by discovery of new mutations. Nat Neurosci 17, 764-772 (2014).

8. M. R. Johnson, K. Shkura, S. R. Langley, A. Delahaye-Duriez, P. Srivastava, W. D. Hill, O. J. Rackham, G. Davies, S. E. Harris, A. Moreno-Moral, M. Rotival, D. Speed, S. Petrovski, A. Katz, C. Hayward, D. J. Porteous, B. H. Smith, S. Padmanabhan, L. J. Hocking, J. M. Starr, D. C. Liewald, A. Visconti, M. Falchi, L. Bottolo, T. Rossetti, B. Danis, M. Mazzuferi, P. Foerch, A. Grote, C. Helmstaedter, A. J. Becker, R. M. Kaminski, I. J. Deary, E. Petretto, Systems genetics identifies a convergent gene network for cognition and neurodevelopmental disease. Nat Neurosci 19, 223-232 (2016).

9. T. Wang, H. Guo, B. Xiong, H. A. Stessman, H. Wu, B. P. Coe, T. N. Turner, Y. Liu, W. Zhao, K. Hoekzema, L. Vives, L. Xia, M. Tang, J. Ou, B. Chen, Y. Shen, G. Xun, M. Long, J. Lin, Z. N. Kronenberg, Y. Peng, T. Bai, H. Li, X. Ke, Z. Hu, J. Zhao, X. Zou, K. Xia, E. E. Eichler, De novo genic mutations among a Chinese autism spectrum disorder cohort. Nature communications 7, 13316 (2016).

10. F. K. Satterstrom, J. A. Kosmicki, J. Wang, M. S. Breen, S. De Rubeis, J. Y. An, M. Peng, R. Collins, J. Grove, L. Klei, C. Stevens, J. Reichert, M. S. Mulhern, M. Artomov, S. Gerges, B. Sheppard, X. Xu, A. Bhaduri, U. Norman, H. Brand, G. Schwartz, R. Nguyen, E. E. Guerrero, C. Dias, C. Autism Sequencing, P.-B. C. i, C. Betancur, E. H. Cook, L. Gallagher, M. Gill, J. S. Sutcliffe, A. Thurm, M. E. Zwick, A. D. Borglum, M. W. State, A. E. Cicek, M. E. Talkowski, D. J. Cutler, B. Devlin, S. J. Sanders, K. Roeder, M. J. Daly, J. D. Buxbaum, Large-Scale Exome Sequencing Study Implicates Both Developmental and Functional Changes in the Neurobiology of Autism. Cell 180, 568584 e523 (2020).

11. M. Wolff, K. M. Johannesen, U. B. S. Hedrich, S. Masnada, G. Rubboli, E. Gardella, G. Lesca, D. Ville, M. Milh, L. Villard, A. Afenjar, S. Chantot-Bastaraud, C. Mignot, C. Lardennois, C. Nava, N. Schwarz, M. Gerard, L. Perrin, D. Doummar, S. Auvin, M. J. Miranda, M. Hempel, E. Brilstra, N. Knoers, N. Verbeek, M. van Kempen, K. P. Braun, G. Mancini, S. Biskup, K. Hortnagel, M. Docker, T. Bast, T. Loddenkemper, L. WongKisiel, F. M. Baumeister, W. Fazeli, P. Striano, R. Dilena, E. Fontana, F. Zara, G. Kurlemann, J. Klepper, J. G. Thoene, D. H. Arndt, N. Deconinck, T. Schmitt-Mechelke, O. Maier, H. Muhle, B. Wical, C. Finetti, R. Bruckner, J. Pietz, G. Golla, D. Jillella, K. M. Linnet, P. Charles, U. Moog, E. Oiglane-Shlik, J. F. Mantovani, K. Park, M. Deprez, D. Lederer, S. Mary, E. Scalais, L. Selim, R. Van Coster, L. Lagae, M. Nikanorova, H. Hjalgrim, G. C. Korenke, M. Trivisano, N. Specchio, B. Ceulemans, T. Dorn, K. L. Helbig, 
798

799

800

801

802

803

804

805

806

807

808

809

810

811

812

K. Hardies, H. Stamberger, P. de Jonghe, S. Weckhuysen, J. R. Lemke, I. KragelohMann, I. Helbig, G. Kluger, H. Lerche, R. S. Moller, Genetic and phenotypic heterogeneity suggest therapeutic implications in SCN2A-related disorders. Brain : a journal of neurology 140, 1316-1336 (2017).

12. M. Wolff, A. Brunklaus, S. M. Zuberi, Phenotypic spectrum and genetics of SCN2Arelated disorders, treatment options, and outcomes in epilepsy and beyond. Epilepsia 60 Suppl 3, S59-S67 (2019).

13. K. Tóth, K. T. Hofer, Á. Kandrács, L. Entz, A. Bagó, L. Erőss, Z. Jordán, G. Nagy, A. Sólyom, D. Fabó, I. Ulbert, L. Wittner, Hyperexcitability of the network contributes to synchronization processes in the human epileptic neocortex. The Journal of physiology 596, 317-342 (2018).

14. R. Planells-Cases, M. Caprini, J. Zhang, E. M. Rockenstein, R. R. Rivera, C. Murre, E. Masliah, M. Montal, Neuronal death and perinatal lethality in voltage-gated sodium channel alpha(II)-deficient mice. Biophys J 78, 2878-2891 (2000).

15. W. Shin, H. Kweon, R. Kang, D. Kim, K. Kim, M. Kang, S. Y. Kim, S. N. Hwang, J. Y. Kim, E. Yang, H. Kim, E. Kim, Scn2a Haploinsufficiency in Mice Suppresses Hippocampal Neuronal Excitability, Excitatory Synaptic Drive, and Long-Term Potentiation, and Spatial Learning and Memory. Frontiers in Molecular Neuroscience 12, (2019).

16. I. Ogiwara, H. Miyamoto, T. Tatsukawa, T. Yamagata, T. Nakayama, N. Atapour, E. Miura, E. Mazaki, S. J. Ernst, D. Cao, H. Ohtani, S. Itohara, Y. Yanagawa, M. Montal, M. Yuzaki, Y. Inoue, T. K. Hensch, J. L. Noebels, K. Yamakawa, Nav1.2 haplodeficiency in excitatory neurons causes absence-like seizures in mice. Commun Biol 1, 96 (2018).

17. H. Miyamoto, T. Tatsukawa, A. Shimohata, T. Yamagata, T. Suzuki, K. Amano, E. Mazaki, M. Raveau, I. Ogiwara, A. Oba-Asaka, T. K. Hensch, S. Itohara, K. Sakimura, K. Kobayashi, K. Kobayashi, K. Yamakawa, Impaired cortico-striatal excitatory transmission triggers epilepsy. Nature communications 10, 1917 (2019).

18. M. V. Fuccillo, Striatal Circuits as a Common Node for Autism Pathophysiology. Front Neurosci 10, 27 (2016).

19. J. Aupy, F. Wendling, K. Taylor, J. Bulacio, J. Gonzalez-Martinez, P. Chauvel, Corticostriatal synchronization in human focal seizures. Brain : a journal of neurology 142, 1282-1295 (2019).

20. P. W. E. Spratt, R. Ben-Shalom, C. M. Keeshen, K. J. Burke, Jr., R. L. Clarkson, S. J. Sanders, K. J. Bender, The Autism-Associated Gene Scn2a Contributes to Dendritic Excitability and Synaptic Function in the Prefrontal Cortex. Neuron 103, 673-685 e675 (2019).

21. M. S. Fallah, J. H. Eubanks, Seizures in Mouse Models of Rare Neurodevelopmental Disorders. Neuroscience 445, 50-68 (2020).

22. M. Eaton, J. Zhang, Z. Ma, A. C. Park, E. Lietzke, C. M. Romero, Y. Liu, E. R. Coleman, X. Chen, T. Xiao, Z. Que, S. Lai, J. Wu, J. H. Lee, S. Palant, H. P. Nguyen, Z. Huang, W. C. Skarnes, W. A. Koss, Y. Yang, Generation and basic characterization of a genetrap knockout mouse model of Scn2a with a substantial reduction of voltage-gated sodium channel Nav 1.2 expression. Genes Brain Behav, e12725 (2020).

23. W. C. Skarnes, H. von Melchner, W. Wurst, G. Hicks, A. S. Nord, T. Cox, S. G. Young, P. Ruiz, P. Soriano, M. Tessier-Lavigne, B. R. Conklin, W. L. Stanford, J. Rossant, C. International Gene Trap, A public gene trap resource for mouse functional genomics. Nat Genet 36, 543-544 (2004).

24. W. C. Skarnes, B. Rosen, A. P. West, M. Koutsourakis, W. Bushell, V. Iyer, A. O. Mujica, M. Thomas, J. Harrow, T. Cox, D. Jackson, J. Severin, P. Biggs, J. Fu, M. Nefedov, P. J. de Jong, A. F. Stewart, A. Bradley, A conditional knockout resource for the genome-wide study of mouse gene function. Nature 474, 337-342 (2011). 
25. W. Hu, B. P. Bean, Differential Control of Axonal and Somatic Resting Potential by Voltage-Dependent Conductances in Cortical Layer 5 Pyramidal Neurons. Neuron 99, 1355 (2018).

26. Z. Huang, M. C. Walker, M. M. Shah, Loss of dendritic HCN1 subunits enhances cortical excitability and epileptogenesis. The Journal of neuroscience : the official journal of the Society for Neuroscience 29, 10979-10988 (2009).

27. G. Testa, J. Schaft, F. van der Hoeven, S. Glaser, K. Anastassiadis, Y. Zhang, T. Hermann, W. Stremmel, A. F. Stewart, A reliable lacZ expression reporter cassette for multipurpose, knockout-first alleles. Genesis 38, 151-158 (2004).

28. K. Y. Chan, M. J. Jang, B. B. Yoo, A. Greenbaum, N. Ravi, W. L. Wu, L. SanchezGuardado, C. Lois, S. K. Mazmanian, B. E. Deverman, V. Gradinaru, Engineered AAVs for efficient noninvasive gene delivery to the central and peripheral nervous systems. Nature neuroscience 20, 1172-1179 (2017).

29. M. H. Meisler, SCN8A encephalopathy: Mechanisms and models. Epilepsia 60 Suppl 3, S86-S91 (2019).

30. R. K. A. Bunton-Stasyshyn, J. L. Wagnon, E. R. Wengert, B. S. Barker, A. Faulkner, P. K. Wagley, K. Bhatia, J. M. Jones, M. R. Maniaci, J. M. Parent, H. P. Goodkin, M. K. Patel, M. H. Meisler, Prominent role of forebrain excitatory neurons in SCN8A encephalopathy. Brain : a journal of neurology 142, 362-375 (2019).

31. L. F. Lopez-Santiago, Y. Yuan, J. L. Wagnon, J. M. Hull, C. R. Frasier, H. A. O'Malley, M. H. Meisler, L. L. Isom, Neuronal hyperexcitability in a mouse model of SCN8A epileptic encephalopathy. Proc Natl Acad Sci U S A 114, 2383-2388 (2017).

32. C. D. Makinson, B. S. Tanaka, J. M. Sorokin, J. C. Wong, C. A. Christian, A. L. Goldin, A. Escayg, J. R. Huguenard, Regulation of Thalamic and Cortical Network Synchrony by Scn8a. Neuron 93, 1165-1179 e1166 (2017).

33. A. V. Vega, D. L. Henry, G. Matthews, Reduced expression of $\mathrm{Na}(\mathrm{v}) 1.6$ sodium channels and compensation by $\mathrm{Na}(\mathrm{v}) 1.2$ channels in mice heterozygous for a null mutation in Scn8a. Neurosci Lett 442, 69-73 (2008).

34. E. Katz, O. Stoler, A. Scheller, Y. Khrapunsky, S. Goebbels, F. Kirchhoff, M. J. Gutnick, F. Wolf, I. A. Fleidervish, Role of sodium channel subtype in action potential generation by neocortical pyramidal neurons. Proceedings of the National Academy of Sciences of the United States of America 115, E7184-E7192 (2018).

35. D. Guan, J. C. Lee, T. Tkatch, D. J. Surmeier, W. E. Armstrong, R. C. Foehring, Expression and biophysical properties of Kv1 channels in supragranular neocortical pyramidal neurones. The Journal of physiology 571, 371-389 (2006).

36. Z. Niday, A. V. Tzingounis, Potassium Channel Gain of Function in Epilepsy: An Unresolved Paradox. The Neuroscientist : a review journal bringing neurobiology, neurology and psychiatry 24, 368-380 (2018).

37. A. Lorincz, Z. Nusser, Cell-type-dependent molecular composition of the axon initial segment. The Journal of neuroscience : the official journal of the Society for Neuroscience 28, 14329-14340 (2008).

38. M. Dumenieu, M. Oule, M. R. Kreutz, J. Lopez-Rojas, The Segregated Expression of Voltage-Gated Potassium and Sodium Channels in Neuronal Membranes: Functional Implications and Regulatory Mechanisms. Front Cell Neurosci 11, 115 (2017).

39. K. Trosclair, H. A. Dhaibar, N. M. Gautier, V. Mishra, E. Glasscock, Neuron-specific Kv1.1 deficiency is sufficient to cause epilepsy, premature death, and cardiorespiratory dysregulation. Neurobiology of disease 137, 104759 (2020).

40. K. Sakamoto, Y. Suzuki, H. Yamamura, S. Ohya, K. Muraki, Y. Imaizumi, Molecular mechanisms underlying pimaric acid-induced modulation of voltage-gated $\mathrm{K}(+)$ channels. J Pharmacol Sci 133, 223-231 (2017). 
863

864

865

866

867

868

869

870

871

872

873

874

875

876

877

878

879

880

881

882

883

884

885

886

887

888

889

890

891

892

893

894

895

896

897

898

899

900

901

902

903

904

905

906

907

908

909

910

911

912

913

41. R. W. Manville, G. W. Abbott, Isoform-Selective KCNA1 Potassium Channel Openers Built from Glycine. J Pharmacol Exp Ther 373, 391-401 (2020).

42. M. Sosa, H. R. Joo, L. M. Frank, Dorsal and Ventral Hippocampal Sharp-Wave Ripples Activate Distinct Nucleus Accumbens Networks. Neuron 105, 725-741.e728 (2020).

43. M. W. Antoine, T. Langberg, P. Schnepel, D. E. Feldman, Increased Excitation-Inhibition Ratio Stabilizes Synapse and Circuit Excitability in Four Autism Mouse Models. Neuron 101, 648-661.e644 (2019).

44. R. E. Westenbroek, J. L. Noebels, W. A. Catterall, Elevated expression of type II Na+ channels in hypomyelinated axons of shiverer mouse brain. J Neurosci 12, 2259-2267 (1992).

45. J. Wang, S. W. Ou, Y. J. Wang, Distribution and function of voltage-gated sodium channels in the nervous system. Channels (Austin) 11, 534-554 (2017).

46. A. M. Rush, S. D. Dib-Hajj, S. G. Waxman, Electrophysiological properties of two axonal sodium channels, Nav1.2 and Nav1.6, expressed in mouse spinal sensory neurones. The Journal of physiology 564, 803-815 (2005).

47. W. Hu, C. Tian, T. Li, M. Yang, H. Hou, Y. Shu, Distinct contributions of $\mathrm{Na}(\mathrm{v}) 1.6$ and $\mathrm{Na}(\mathrm{v}) 1.2$ in action potential initiation and backpropagation. Nat Neurosci 12, 996-1002 (2009).

48. A. D. Workman, C. J. Charvet, B. Clancy, R. B. Darlington, B. L. Finlay, Modeling transformations of neurodevelopmental sequences across mammalian species. The Journal of neuroscience : the official journal of the Society for Neuroscience 33, 73687383 (2013).

49. E. V. Gazina, K. L. Richards, M. B. Mokhtar, E. A. Thomas, C. A. Reid, S. Petrou, Differential expression of exon 5 splice variants of sodium channel alpha subunit mRNAs in the developing mouse brain. Neuroscience 166, 195-200 (2010).

50. K. J. Bender, L. O. Trussell, The physiology of the axon initial segment. Annual review of neuroscience 35, 249-265 (2012).

51. U. B. S. Hedrich, S. Lauxmann, H. Lerche, SCN2A channelopathies: Mechanisms and models. Epilepsia 60 Suppl 3, S68-S76 (2019).

52. R. Ben-Shalom, C. M. Keeshen, K. N. Berrios, J. Y. An, S. J. Sanders, K. J. Bender, Opposing Effects on NaV1.2 Function Underlie Differences Between SCN2A Variants Observed in Individuals With Autism Spectrum Disorder or Infantile Seizures. Biol Psychiatry 82, 224-232 (2017).

53. I. H. Quraishi, S. Stern, K. P. Mangan, Y. Zhang, S. R. Ali, M. R. Mercier, M. C. Marchetto, M. J. McLachlan, E. M. Jones, F. H. Gage, L. K. Kaczmarek, An EpilepsyAssociated KCNT1 Mutation Enhances Excitability of Human iPSC-Derived Neurons by Increasing Slack KNa Currents. The Journal of neuroscience : the official journal of the Society for Neuroscience 39, 7438-7449 (2019).

54. H. Soh, S. Park, K. Ryan, K. Springer, A. Maheshwari, A. V. Tzingounis, Deletion of $\mathrm{KCNQ} 2 / 3$ potassium channels from $\mathrm{PV}$ + interneurons leads to homeostatic potentiation of excitatory transmission. eLife 7, (2018).

55. Z. Niday, V. E. Hawkins, H. Soh, D. K. Mulkey, A. V. Tzingounis, Epilepsy-Associated KCNQ2 Channels Regulate Multiple Intrinsic Properties of Layer 2/3 Pyramidal Neurons. The Journal of neuroscience : the official journal of the Society for Neuroscience 37, 576-586 (2017).

56. J. J. Millichap, K. L. Park, T. Tsuchida, B. Ben-Zeev, L. Carmant, R. Flamini, N. Joshi, P. M. Levisohn, E. Marsh, S. Nangia, V. Narayanan, X. R. Ortiz-Gonzalez, M. C. Patterson, P. L. Pearl, B. Porter, K. Ramsey, E. L. McGinnis, M. Taglialatela, M. Tracy, B. Tran, C. Venkatesan, S. Weckhuysen, E. C. Cooper, KCNQ2 encephalopathy: Features, mutational hot spots, and ezogabine treatment of 11 patients. Neurology. Genetics 2 , e96 (2016). 
57. I. H. Quraishi, M. R. Mercier, H. McClure, R. L. Couture, M. L. Schwartz, R. Lukowski, P. Ruth, L. K. Kaczmarek, Impaired motor skill learning and altered seizure susceptibility in mice with loss or gain of function of the Kcnt1 gene encoding Slack (KNa1.1) $\mathrm{Na}(+)$ activated K(+) channels. Sci Rep 10, 3213 (2020).

58. J. Johnston, I. D. Forsythe, C. Kopp-Scheinpflug, SYMPOSIUM REVIEW: Going native: voltage-gated potassium channels controlling neuronal excitability. The Journal of physiology 588, 3187-3200 (2010).

59. H. Wulff, N. A. Castle, L. A. Pardo, Voltage-gated potassium channels as therapeutic targets. Nature Reviews Drug Discovery 8, 982-1001 (2009).

60. R. C. Wykes, J. H. Heeroma, L. Mantoan, K. Zheng, D. C. MacDonald, K. Deisseroth, K. S. Hashemi, M. C. Walker, S. Schorge, D. M. Kullmann, Optogenetic and potassium channel gene therapy in a rodent model of focal neocortical epilepsy. Sci Trans/ Med 4, 161 ra152 (2012).

61. A. Snowball, E. Chabrol, R. C. Wykes, T. Shekh-Ahmad, J. H. Cornford, A. Lieb, M. P. Hughes, G. Massaro, A. A. Rahim, K. S. Hashemi, D. M. Kullmann, M. C. Walker, S. Schorge, Epilepsy Gene Therapy Using an Engineered Potassium Channel. The Journal of neuroscience : the official journal of the Society for Neuroscience 39, 3159-3169 (2019).

62. A. T. Berg, H. Palac, G. Wilkening, F. Zelko, L. Schust Meyer, SCN2A-Developmental and Epileptic Encephalopathies: Challenges to trial-readiness for non-seizure outcomes. Epilepsia, (2020).

63. L. E. Fenno, J. Mattis, C. Ramakrishnan, M. Hyun, S. Y. Lee, M. He, J. Tucciarone, A. Selimbeyoglu, A. Berndt, L. Grosenick, K. A. Zalocusky, H. Bernstein, H. Swanson, C. Perry, I. Diester, F. M. Boyce, C. E. Bass, R. Neve, Z. J. Huang, K. Deisseroth, Targeting cells with single vectors using multiple-feature Boolean logic. Nat Methods 11, 763-772 (2014).

64. L. D. Liu, S. Chen, M. N. Economo, N. Li, K. Svoboda, Accurate localization of linear probe electrodes across multiple brains. bioRxiv, 2020.2002.2025.965210 (2020).

65. J. Wang, X. He, H. Meng, Y. Li, P. Dmitriev, F. Tian, J. C. Page, Q. R. Lu, Z. He, Robust Myelination of Regenerated Axons Induced by Combined Manipulations of GPR17 and Microglia. Neuron 108, 876-886.e874 (2020).

66. A. M. Bolger, M. Lohse, B. Usadel, Trimmomatic: a flexible trimmer for Illumina sequence data. Bioinformatics 30, 2114-2120 (2014).

67. S. Andrews, FastQC. (2010).

68. A. Dobin, C. A. Davis, F. Schlesinger, J. Drenkow, C. Zaleski, S. Jha, P. Batut, M. Chaisson, T. R. Gingeras, STAR: ultrafast universal RNA-seq aligner. Bioinformatics 29, 15-21 (2013).

69. S. Anders, P. T. Pyl, W. Huber, HTSeq--a Python framework to work with highthroughput sequencing data. Bioinformatics 31, 166-169 (2015).

70. K. J. Livak, T. D. Schmittgen, Analysis of relative gene expression data using real-time quantitative PCR and the 2(-Delta Delta C(T)) Method. Methods 25, $402-408$ (2001).

71. M. E. Torres-Garcia, O. Solis, A. Patricio, A. Rodriguez-Moreno, I. Camacho-Abrego, I. D. Limon, G. Flores, Dendritic morphology changes in neurons from the prefrontal cortex, hippocampus and nucleus accumbens in rats after lesion of the thalamic reticular nucleus. Neuroscience 223, 429-438 (2012).

72. R. L. Clarkson, A. T. Liptak, S. M. Gee, V. S. Sohal, K. J. Bender, D3 Receptors Regulate Excitability in a Unique Class of Prefrontal Pyramidal Cells. The Journal of Neuroscience 37, 5846-5860 (2017).

73. N. C. Dembrow, R. A. Chitwood, D. Johnston, Projection-Specific Neuromodulation of Medial Prefrontal Cortex Neurons. The Journal of Neuroscience 30, 16922-16937 (2010). 
74. M. J. Fogarty, L. A. Hammond, R. Kanjhan, M. C. Bellingham, P. G. Noakes, A method for the three-dimensional reconstruction of Neurobiotin-filled neurons and the location of their synaptic inputs. Frontiers in neural circuits 7, 153 (2013).

75. X. Jia, J. H. Siegle, C. Bennett, S. D. Gale, D. J. Denman, C. Koch, S. R. Olsen, Highdensity extracellular probes reveal dendritic backpropagation and facilitate neuron classification. Journal of neurophysiology 121, 1831-1847 (2019).

76. J. H. Siegle, X. Jia, S. Durand, S. Gale, C. Bennett, N. Graddis, G. Heller, T. K. Ramirez, H. Choi, J. A. Luviano, P. A. Groblewski, R. Ahmed, A. Arkhipov, A. Bernard, Y. N. Billeh, D. Brown, M. A. Buice, N. Cain, S. Caldejon, L. Casal, A. Cho, M. Chvilicek, T. C. Cox, K. Dai, D. J. Denman, S. E. J. de Vries, R. Dietzman, L. Esposito, C. Farrell, D. Feng, J. Galbraith, M. Garrett, E. C. Gelfand, N. Hancock, J. A. Harris, R. Howard, B. Hu, R. Hytnen, R. Iyer, E. Jessett, K. Johnson, I. Kato, J. Kiggins, S. Lambert, J. Lecoq, P. Ledochowitsch, J. H. Lee, A. Leon, Y. Li, E. Liang, F. Long, K. Mace, J. Melchior, D. Millman, T. Mollenkopf, C. Nayan, L. Ng, K. Ngo, T. Nguyen, P. R. Nicovich, K. North, G. K. Ocker, D. Ollerenshaw, M. Oliver, M. Pachitariu, J. Perkins, M. Reding, D. Reid, M. Robertson, K. Ronellenfitch, S. Seid, C. Slaughterbeck, M. Stoecklin, D. Sullivan, B. Sutton, J. Swapp, C. Thompson, K. Turner, W. Wakeman, J. D. Whitesell, D. Williams, A. Williford, R. Young, H. Zeng, S. Naylor, J. W. Phillips, R. C. Reid, S. Mihalas, S. R. Olsen, $\mathrm{C}$. Koch, A survey of spiking activity reveals a functional hierarchy of mouse corticothalamic visual areas. bioRxiv, 805010 (2019).

\section{ACKNOWLEDGMENTS}

General: We thank Dr. Amy Brewster and Dr. Chongli Yuan for the critical reading of this paper.

Funding: This work is supported by Purdue startup funding, Ralph W. and Grace M. Showalter Research Trust, and Purdue Big Idea Challenge 2.0 on Autism to Y.Y. The authors gratefully acknowledge support from the Purdue University Institute for Drug Discovery and Institute for Integrative Neuroscience. M.E. is supported by the National Science Foundation (NSF) Graduate Research Fellowship Program (GRFP) (DGE1842166). Yang lab is grateful to the FamilieSCN2A foundation for the Action Potential Grant support. This project was funded, in part, with support from the Indiana Clinical and Translational Sciences Institute funded, in part by Award Number UL1TR002529 from the National Institutes of Health, National Center for Advancing Translational Sciences, Clinical and Translational Sciences Award. The Yang lab appreciates the bioinformatics support of the Collaborative Core for Cancer Bioinformatics $\left(C^{3} B\right)$ with support from the Indiana University Simon Comprehensive Cancer Center (Grant P30CA082709), Purdue University Center for Cancer Research (Grant P30CA023168), and Walther Cancer Foundation. The content is solely the responsibility of the authors and does not necessarily represent the official views of the National Institutes of Health.

Author contributions: J.Z., X.C., Y.Y. designed the experiments. J.Z., X.C., M.E., S.L., A.P., T.S.A., J.W., Z.M., Z.Q., J.L., T.X., Y.L., Y.W., M.I.O.A., N.A.L. performed the experiments and analyzed the data. J.A.S, K.J., Z.H., N.A.L., W.C.S. participate in data 
1009 analysis and experimental design. Y.Y. supervised the project. J.Z. and Y.Y. wrote the 1010 paper with inputs from all authors.

1011

1012 Competing interests: The authors declare no competing interests.

1013

1014 Data and materials availability: Additional results can be found in the supplemental 1015 material. The data that supports the findings of this study are available from the 1016 corresponding author upon reasonable request. 
A

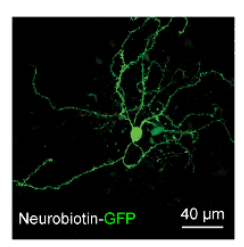

B

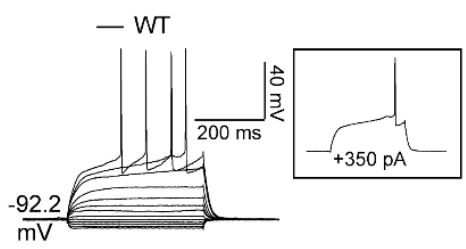

E(i)

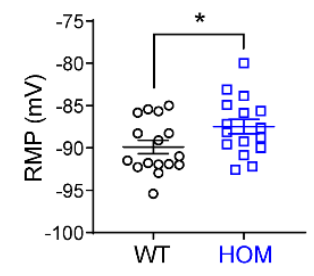

$\mathrm{H}$

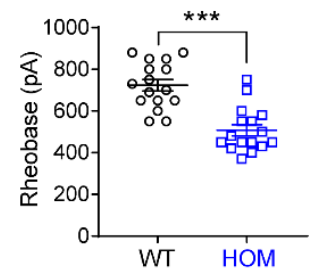

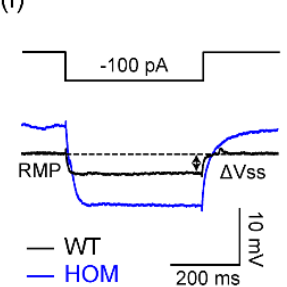

I

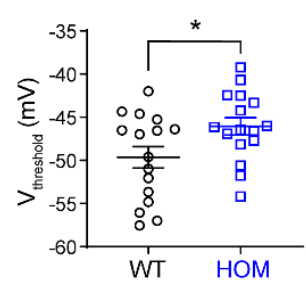

E(ii)

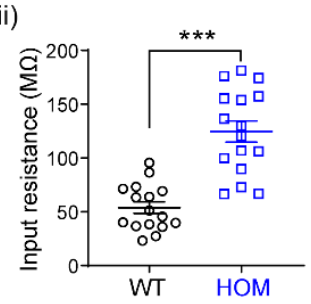

J

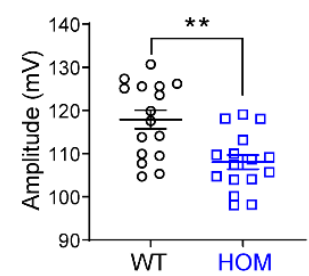

- HOM

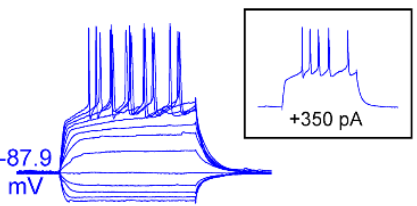

F

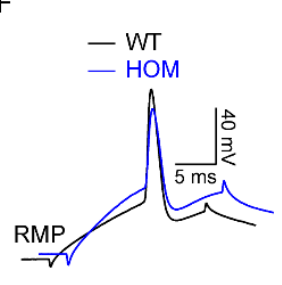

K

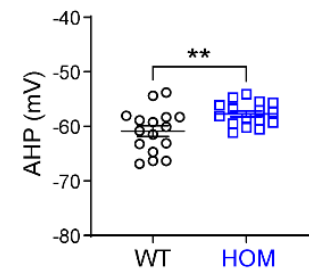

C

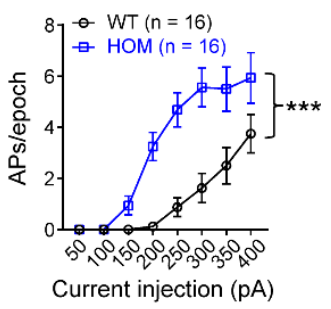

G
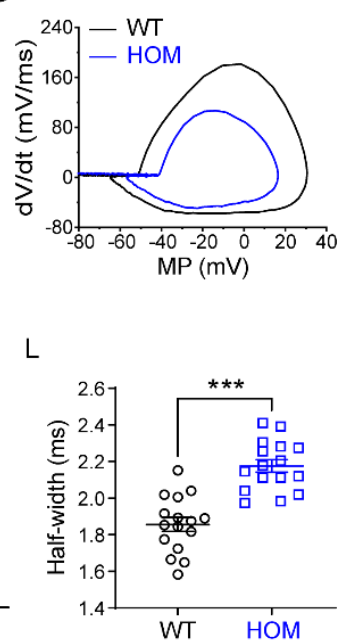

1021

1022

1023

1024

1025

1026

1027

1028

1029

1030

1031

1032

1033

1034

1035

1036

1037

Fig. 1. Elevated neuronal firings of striatal medium spiny neurons (MSNs) in adult Nav1.2deficient mice.

(A) A typical MSN labeled by neurobiotin. Scale bar, $40 \mu \mathrm{m}$. (B) Representative current-clamp recordings of MSNs from WT (black) and homozygous (HOM), Scn2atko/gtKo (blue) mice were obtained at the resting membrane potential (RMP). A series of 400-ms hyperpolarizing and depolarizing steps in 50-pA increments were applied to produce the traces. Inset: representative trace in response to $350 \mathrm{pA}$ positive current injection. (C) The average number of action potentials (APs) generated in response to depolarizing current pulses. Unpaired two-tailed non-parametric Mann-Whitney U-test for each current pulse: ${ }^{* *} p<0.001$. (D) Individuals and mean RMP values. Unpaired two-tailed Student's $t$-test: ${ }^{*} \mathrm{p}<0.05$. (Ei) Representative traces in response to $100 \mathrm{pA}$ negative current injection. $V_{\text {steady-state }}\left(V_{s s}\right)$ is the voltage recorded at $0-10 \mathrm{~ms}$ before the end of the stimulus. (Eii) Individuals and mean input resistance values at the RMP. Unpaired two-tailed Student's $t$-test: ${ }^{* * *} p<0.001$. (F) Typical spikes of MSNs from WT (black) and HOM (blue) mice were obtained at the normal RMP. (G) Associated phase-plane plots. (H-L) Individuals and mean spike rheobase, voltage threshold, amplitude, fAHP (fast after-hyperpolarization), and half-width values. Unpaired two-tailed Student's $t$-test: ${ }^{*} p<0.05$; ${ }^{* *} p<0.01$; ${ }^{* *} p<0.001$. Data were shown as mean $\pm \mathrm{SEM}$. 
Figure 2

A

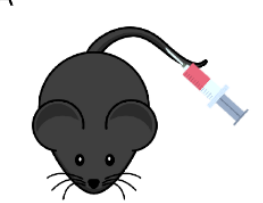

Tail vein injection: AAV-PHP.eB.Control AAV-PHP.eB.FIpO

D

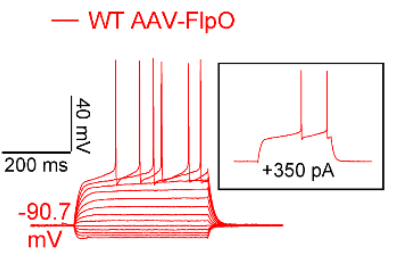
- HOM AAV-Control

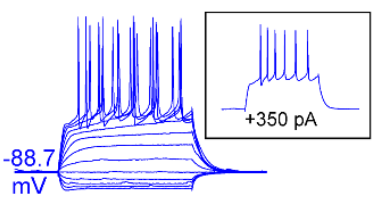

F

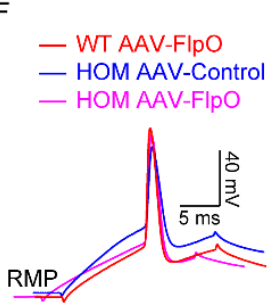

B

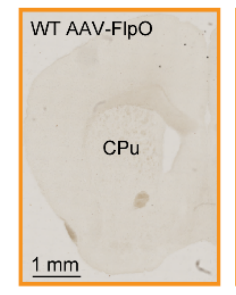

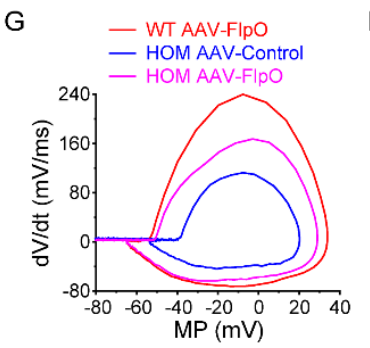

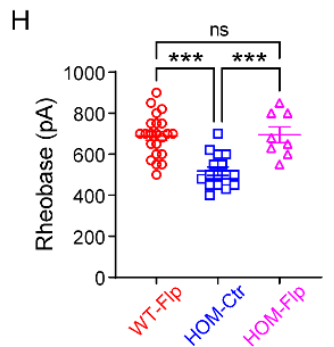
- HOM AAV-FIpO
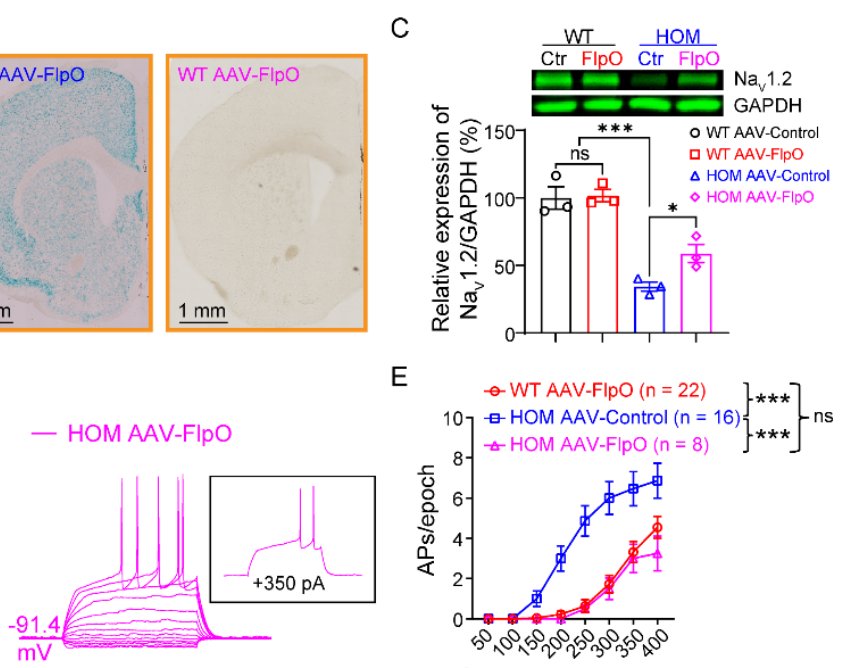

E
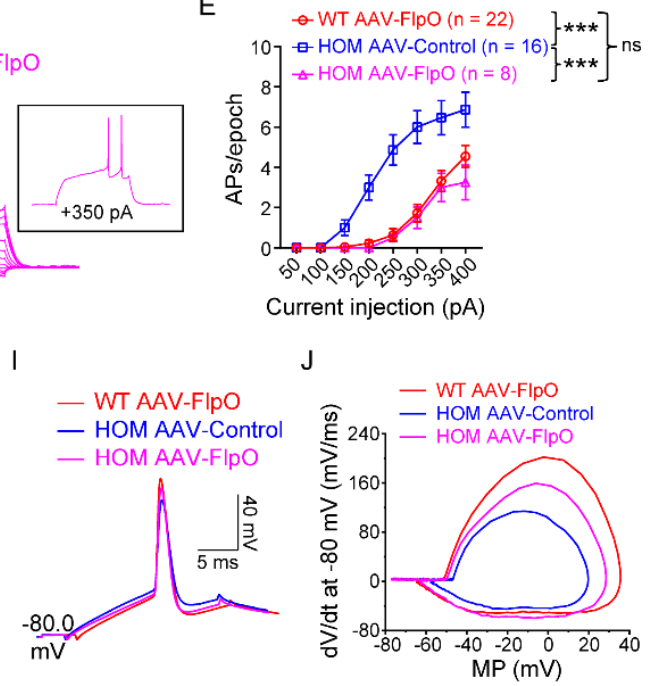

1040

1041

1042

1043

1044

1045

1046

1047

1048

1049

1050

1051

1052

1053

1054

1055

1056

1057

1058

1059

1060

1061

1062

1063

1064

Fig. 2. Elevated neuronal firing is reversible by FlpO-mediated restoration of Nav1.2 expression in adult $\mathrm{Na}_{v} 1.2-$ deficient mice.

(A) Cartoon illustration of mice systemically administrated with PHP.eB.AAV-control or AAV-FlpO via tail vein injection. (B) Coronal views of $L a c Z$ staining of striatum from WT and Scn2a $a^{\text {gtKO/gtko }}$ $(\mathrm{HOM})$ mice injected with AAV-control or AAV-FlpO. Blue staining of HOM mice largely disappeared in the AAV-FlpO group. CPu, caudate nucleus and the putamen (dorsal striatum). (C) The Western blot analysis showed Nav1.2 protein levels in whole-brain tissues from Scn2a $a^{\text {gtKO/gtKo }}(\mathrm{HOM})$ mice in AAV-Control or AAV-FlpO group. One-way ANOVA with Bonferroni's multiple-comparison test: ns, no significance, $p>0.05$; ${ }^{*} p<0.05$; ${ }^{* \star} p<0.001$. (D) Representative current-clamp recordings of MSNs from WT mice transduced with AAV-FIpO (red), HOM mice transduced with AAV-Control (blue), and HOM mice transduced with AAV-Control (magenta) obtained at the RMP. A series of 400-ms hyperpolarizing and depolarizing steps in 50-pA increments were applied to produce the traces. Inset: representative trace in response to $350 \mathrm{pA}$ positive current injection. (E) The average number of APs generated in response to depolarizing current pulses at the RMP. Unpaired two-tailed non-parametric Mann-Whitney U-test for each current pulse: ns, no significance, ${ }^{*} p>0.05 ;{ }^{* * *} p<0.001$. (F) Typical spikes of MSNs from WT transduced with AAV-FlpO (red), HOM transduced with AAV-Control (blue) and HOM transduced with AAV-Control (magenta) were obtained at the normal RMP. (G) Associated phase-plane plots. (H) Individuals and average spike rheobase. Unpaired two-tailed Student's $t$-test: ns, no significance, $p>0.05 ;{ }^{* * *} p<0.001$. (I) Typical spikes of MSNs from WT mice transduced with AAV-FlpO (red), HOM mice transduced with AAV-Control (blue), and HOM mice transduced with AAV-Control (magenta) at a fixed membrane potential of $-80 \mathrm{mV}$. (J) Associated phase-plane plots at $-80 \mathrm{mV}$. Data were shown as mean \pm SEM. 
Figure 3

A Sparse AAV-FIpO transduction

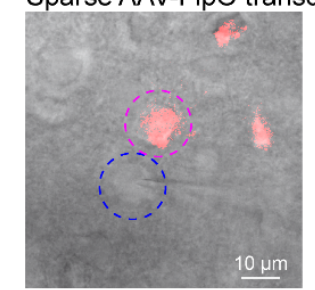

C

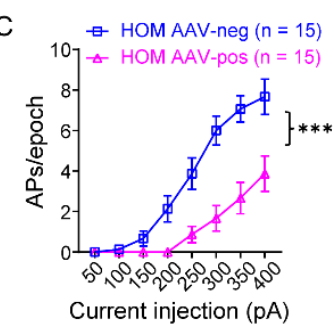

$\mathrm{H}$

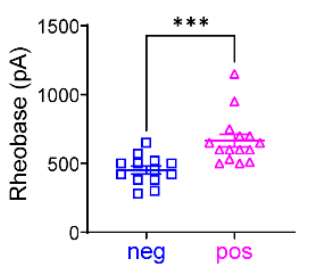

I
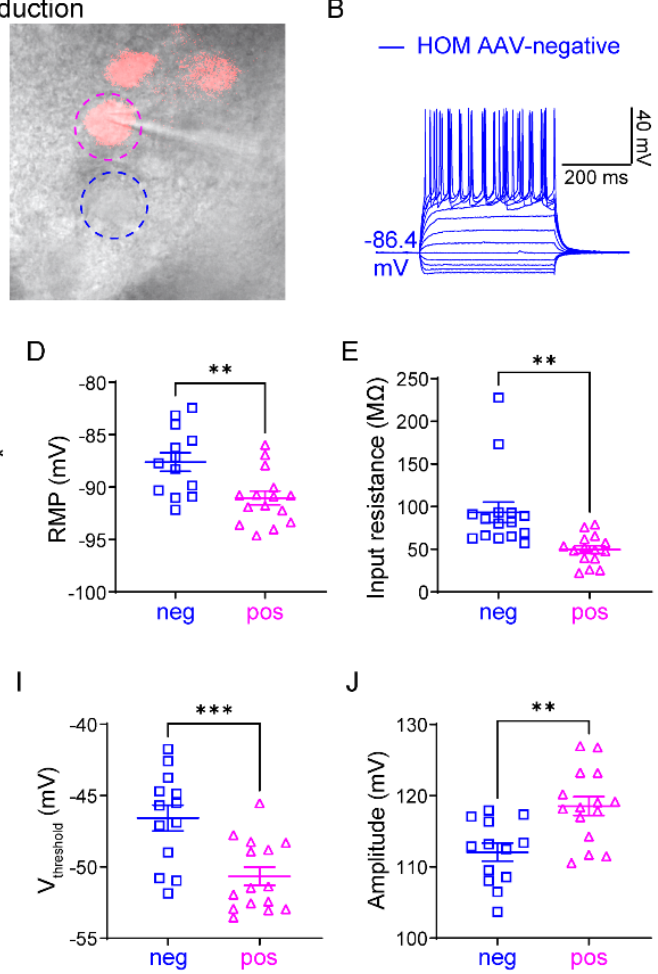

$\mathrm{J}$

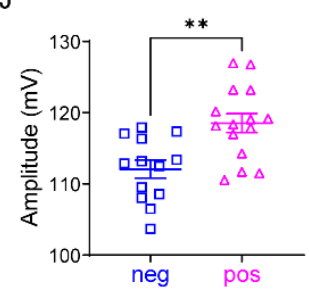

B - HOM AAV-negative

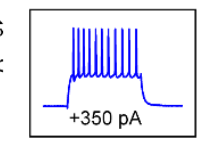
- HOM AAV-positive
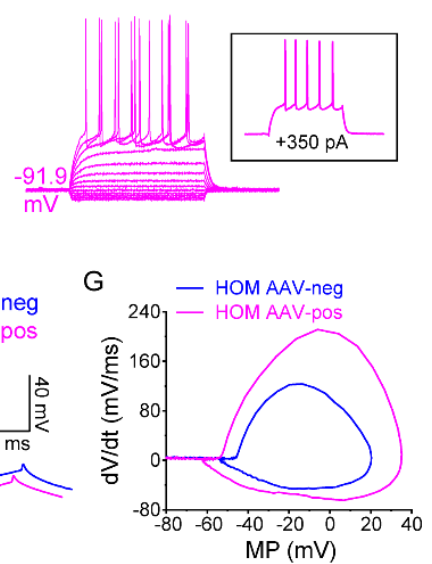

K

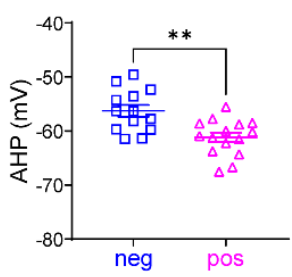

L

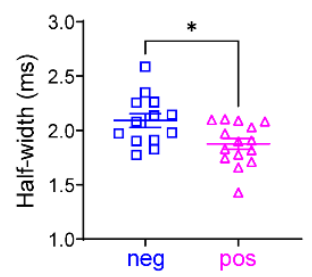

Fig. 3. Elevated neuronal excitability is autonomous.

1068

1069

1070

1071

1072

1073

1074

1075

1076

1077

1078

1079

1080

1081

1082

1083

(A) Scn2a $a^{g t K O / g t K o}(\mathrm{HOM})$ mice were injected with a dilute FlpO virus, transducing a subset of neurons in the striatum sparsely. Dashed circles highlight two neighboring AAV-negative (blue circle) and AAV-FlpO-positive (magenta circle) neurons. The images were taken in the cellattached configuration, and after that, the target neurons were used for whole-cell recordings. (B) Representative current-clamp recordings of AAV-negative (blue) and AAV-FlpO-positive (magenta) MSNs in CPu of Scn2a $a^{g t K O / g t K O}$ mice were obtained at the RMP. A series of 400-ms hyperpolarizing and depolarizing steps in 50-pA increments were applied to produce the traces. Inset: representative trace in response to $350 \mathrm{pA}$ positive current injection. (C) The average number of APs generated in response to depolarizing current pulses. Unpaired two-tailed nonparametric Mann-Whitney U-test for each current pulse: ${ }^{* *} p<0.001$. (D) Individuals and average RMP values. Unpaired two-tailed Student's $t$-test: ${ }^{* *} p<0.01$. (E) Individuals and average input resistance values at the RMP. Unpaired two-tailed Student's $t$-test: ${ }^{* *} p<0.01$. (F) Typical spikes of MSNs with AAV-negative (blue) and with AAV-FIpO-positive (magenta) in HOM mice were obtained at the RMP. (G) Associated phase-plane plots. (H-L) Individuals and average spike rheobase, voltage threshold, amplitude, fAHP, and half-width values. Unpaired two-tailed Student's $t$-test: ${ }^{*} p<0.05 ;{ }^{* *} p<0.01 ;{ }^{* * *} p<0.001$. Data were shown as mean \pm SEM. 
Figure 4

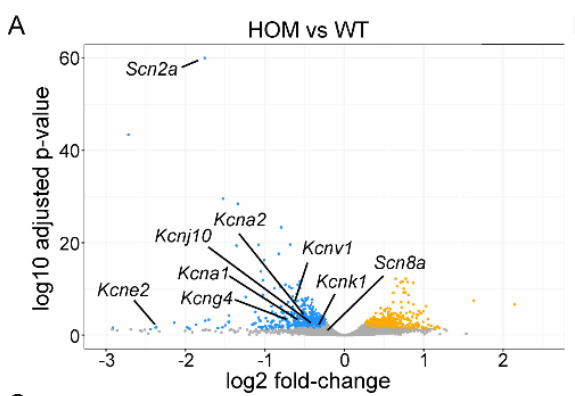

C - WT Control

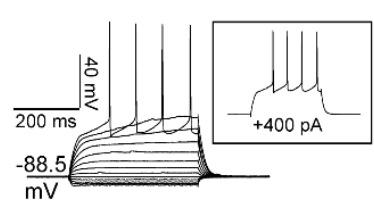

- HOM Control

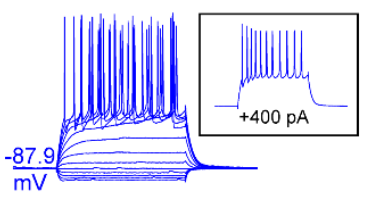
B

\begin{tabular}{|c|c|c|}
\hline \% expression & Gene name & Gene description \\
\hline 18.42 & Kcne2 & potassium voltage-gated channel, Isk-related subfamily, gene 2 (regulatory) \\
\hline 59.49 & Kcng4 & potassium voltage-gated channel, subfamily $\mathrm{G}$, member 4 (modifier) \\
\hline 65.05 & Konv1 & potassium channel, subfamily V, member 1 (modifier) \\
\hline 66.93 & Kcna1 & potassium voltage-gated channel, shaker-related subfamily, member $1\left(K_{v} 1.1\right)$ \\
\hline 70.25 & Kcna2 & potassium voltage-gated channel, shaker-related subfamily, member $2\left(\mathrm{~K}_{v} 1.2\right)$ \\
\hline 75.00 & Kcnj10 & potassium inwardly-rectifying channel, subfamily $\mathrm{J}$, member 10 \\
\hline 80.79 & Konk1 & potassium channel, subfamily $\mathrm{K}$, member 1 (two pore domain) \\
\hline 29.57 & Scn2a & sodium channel, voltage-gated, type II, alpha $\left(\mathrm{Na}_{\mathrm{v}} 1.2\right)$ \\
\hline 80.79 & Scn8a & sodium channel, voltage-gated, type $\mathrm{VIII}$, alpha $\left(\mathrm{Na}_{\mathrm{v}} 1.6\right)$ \\
\hline
\end{tabular}

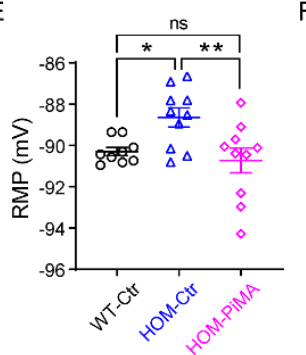

J

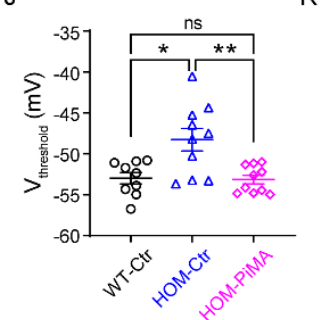

F
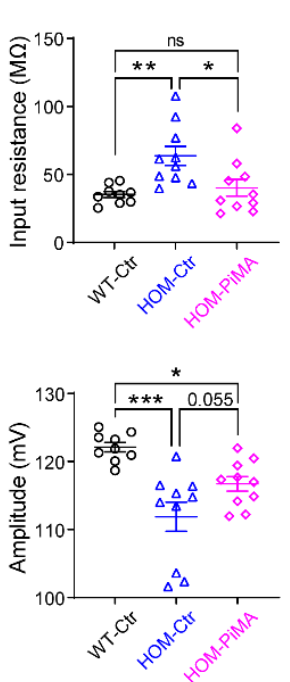

- WT Control
- HOM Control

- HOM $10 \mu \mathrm{M}$ PiMA

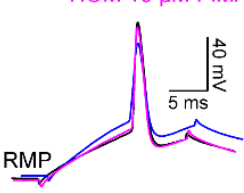

L

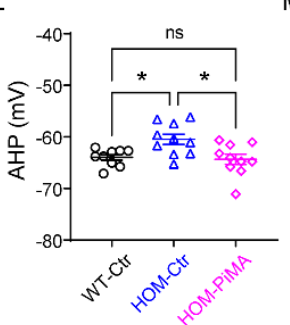

- HOM $10 \mu \mathrm{M}$ PIMA

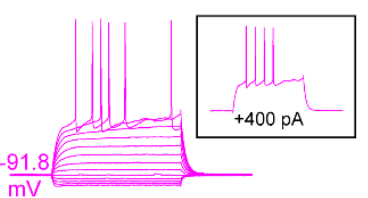

H
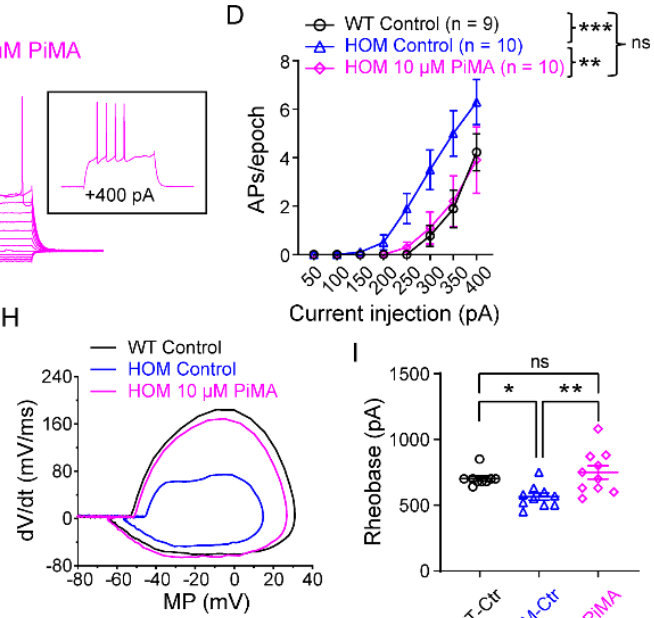

Fig. 4. Activation of $K_{v}$ channels reverses elevated neuronal firings in adult Nav1.2deficient mice.

(A) Volcano plot displays Scn2a and Scn8a, as well all potassium channels that are statistically down-regulated in Scn2atKo/gtKo $(\mathrm{HOM})$ mice compared to WT mice identified by RNA-seq. Statistically significantly upregulated genes are shown in yellow and downregulated genes are shown in blue. (B) List of potassium channels that are significantly down-regulated in HOM mice compared to WT. (Hits that are identified from both DESeq2 and edgeR differential expression analysis with False Discovery Rate < 0.05 were listed). "\% expression”: percentage expression of the gene in HOM mice considering the value of WT mice as $100 \%$. ( $n=4$ mice for each group). (C) Representative current-clamp recordings of MSNs from WT slices perfused with $0.1 \%$ DMSO in aCSF (WT Control, black), HOM slices perfused with 0.1\% DMSO in aCSF (HOM Control, blue), and HOM slices perfused with $0.1 \%$ DMSO in aCSF containing PiMA (HOM $10 \mu M$ PiMA, magenta) at the RMP. A series of 400-ms hyperpolarizing and depolarizing steps in 50-pA increments were applied to produce the traces. Inset: representative trace in response to $400 \mathrm{pA}$ positive current injection. (D) The average number of APs generated in response to depolarizing current pulses at the RMP. Unpaired two-tailed non-parametric Mann-Whitney U-test for each current pulse: ns, no significance, ${ }^{*} p>0.05 ;{ }^{* *} p<0.01 ;{ }^{* * *} p<0.001$. (E) Individuals and average 
RMP values. Unpaired two-tailed Student's $t$-test: ns, no significance, ${ }^{*} \mathrm{p}>0.05 ;{ }^{*} \mathrm{p}<0.05 ;{ }^{* *} \mathrm{p}<$ 0.01. (F) Individuals and average input resistance values at the RMP. Unpaired two-tailed Student's $t$-test: ns, no significance, ${ }^{*} p>0.05$; ${ }^{*} p<0.05 ;{ }^{* *} p<0.01$. (G) Typical spikes of MSNs from WT slices perfused with $0.1 \%$ DMSO in aCSF (WT Control, black), HOM slices perfused with $0.1 \%$ DMSO in aCSF (HOM Control, blue), and HOM slices perfused with $0.1 \%$ DMSO in aCSF containing PiMA (HOM $10 \mu \mathrm{M}$ PiMA, magenta) were obtained at the RMP. (H) Associated phaseplane plots. (I-M) Individuals and average spike rheobase, voltage threshold, amplitude, fAHP, and half-width values. Unpaired two-tailed Student's $t$-test: ns, no significance, ${ }^{*} p>0.05$; ${ }^{*} p<$ $0.05 ;{ }^{* *} p<0.01 ;{ }^{* \star *} p<0.001$. Data were shown as mean \pm SEM. 


\section{SUPPLEMENTARY MATERIALS}

\section{Supplementary Figure 1}

A Tm1a (knockout first allele, gene trap knockout/gtKO)

Trapped splicing

Skipping over (full length functional) transcript

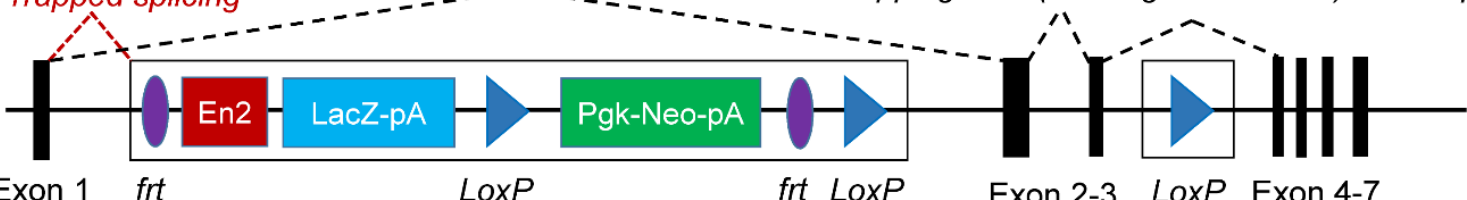

Flp

B

Tm1c (conditional/rescue allele)

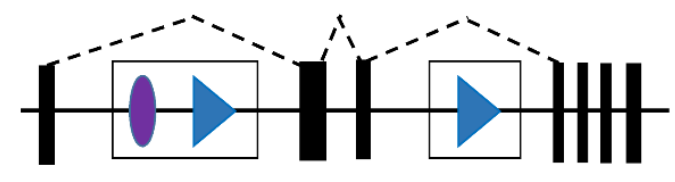

Exon 1 frt LoxP Exon 2-3 LoxP Exon 4-7

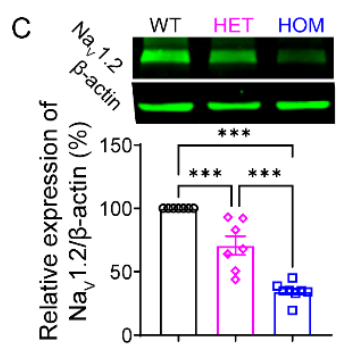

D

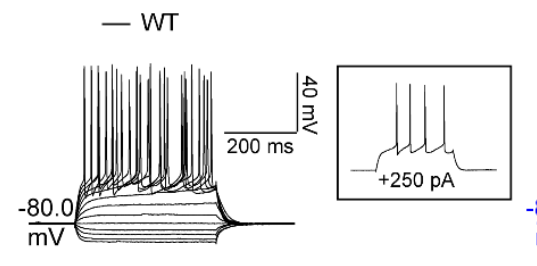

$\mathrm{F}(\mathrm{i})$

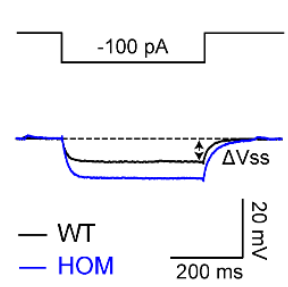

I

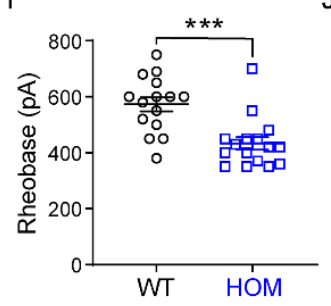

$\mathrm{F}$ (ii)

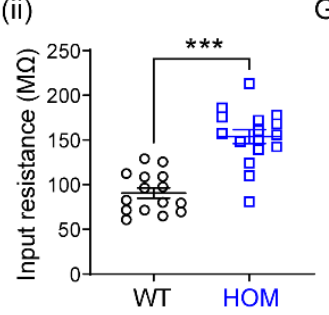

$J$

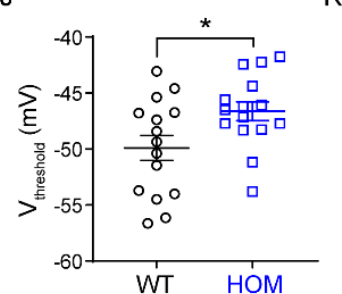

G

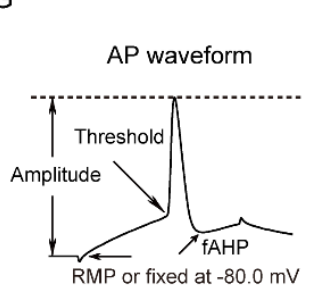

K

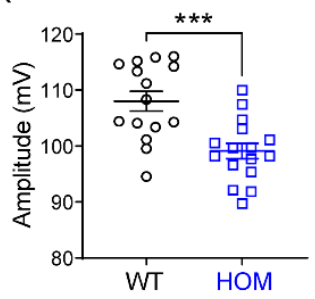

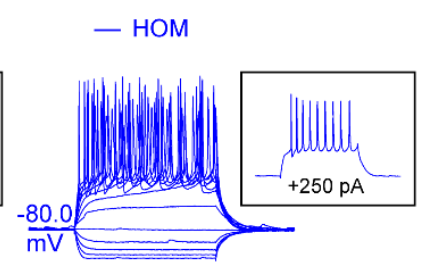

E
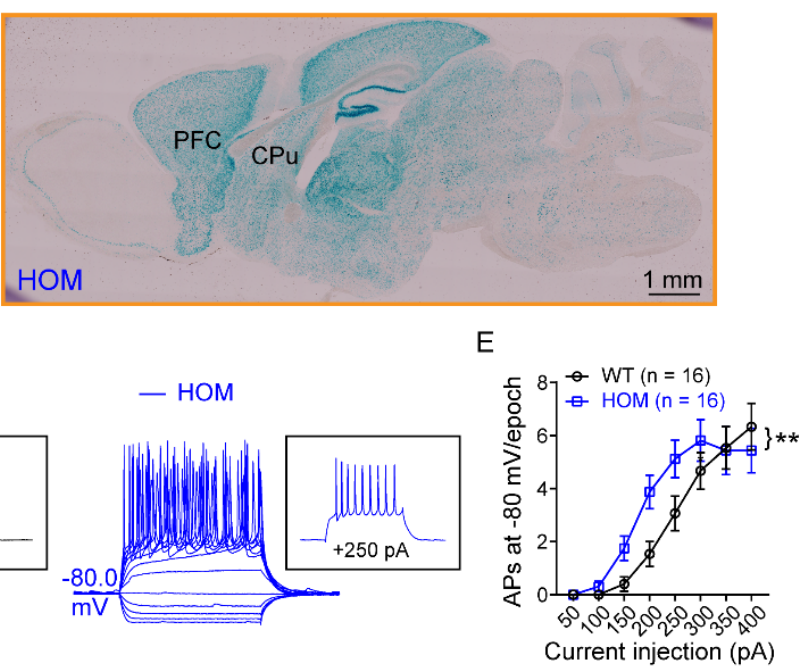

Figure S1. Elevated neuronal firings of striatal MSNs at a fixed membrane potential of -80 $\mathrm{mV}$ in adult Nav1.2-deficient mice. Related to Figure 1.

(A) gtKO allele has an inserted tm1a trapping cassette between the Exon 1 and Exon 2 of Scn2a gene in the genome, which traps the transcription from Exon 1 to tm1a cassette, resulting in "gene-trap" knockout of Scn2a. In the presence of Flp recombinase, frt sites flanked trapping cassette will be removed, producing conditional ("rescue") allele that allows the expression of Scn2a at the WT level. frt, Flp recognition target (purple); En2, engrailed-2 splice acceptor (red); LacZ, lacZ $\beta$-galactosidase (light blue); LoxP, locus of X-over P1 (dark blue); and Neo, neomycin 
(green). (B) gtKO cassette contains a LacZ element and is driven by the native Scn2a promoter. Thus, the LacZ expression can be used as a surrogate of Scn2a expression. Representative LacZ staining of a sagittal slice from a Scn2a $a^{g t K O / g t K O}(\mathrm{HOM})$ mouse showing a strong blue signal across the brain including the prefrontal cortex (PFC) and dorsal striatum (CPu, caudate nucleus and the putamen). (C) Upper: Representative Western blots of striatal tissues from WT (black circle), HET (magenta diamond), and HOM (blue square) mice. Lower: associated quantification of Nav1.2 protein. One-way ANOVA followed by Tukey's multiple-comparison test: ${ }^{* *} p<0.001$. (D) Representative current-clamp recordings of MSNs from WT (black) and HOM (blue) mice were obtained at a fixed membrane potential of $-80 \mathrm{mV}$. A series of $400-\mathrm{ms}$ hyperpolarizing and depolarizing steps in 50-pA increments were applied to produce the traces. Inset: representative trace in response to $250 \mathrm{pA}$ positive current injection. (E) The average number of APs generated in response to depolarizing current pulses at $-80 \mathrm{mV}$. Unpaired two-tailed non-parametric MannWhitney U-test for each current pulse: ${ }^{* *} p<0.01$. (Fi) Representative traces in response to 100 pA negative current injection. $V_{\text {steady-state }}\left(V_{s s}\right)$ is the voltage recorded at $0-10 \mathrm{~ms}$ before the end of the stimulus. (Fii) Individuals and average input resistance values at $-80 \mathrm{mV}$. Unpaired two-tailed Student's $t$-test: ${ }^{* * *} p<0.001$. (G) Left: plot of a typical AP showed its various phases. Right: typical spikes of MSNs from WT (black) and HOM (blue) mice were obtained at a fixed membrane potential of $-80 \mathrm{mV}$. (H) Associated phase-plane plots. (I-M) Individuals and average spike rheobase, voltage threshold, amplitude, fAHP (fast after-hyperpolarization), and half-width values. 


\section{Supplementary Figure 2}

A

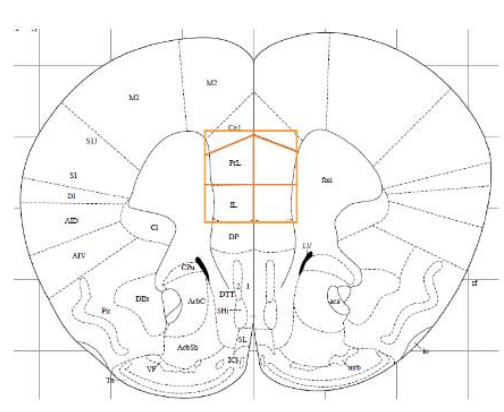

D

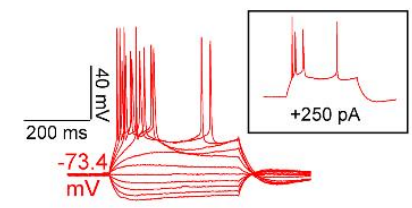

$\mathrm{F}$
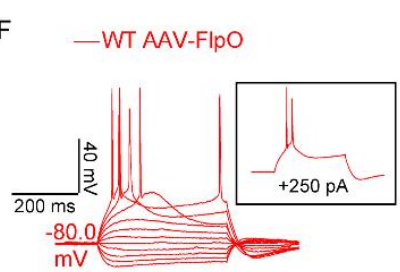

- HOM AAV-Control

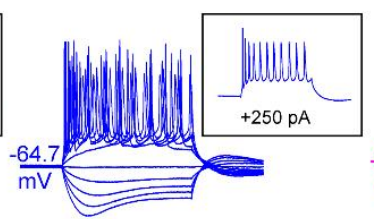

- HOM AAV-Control

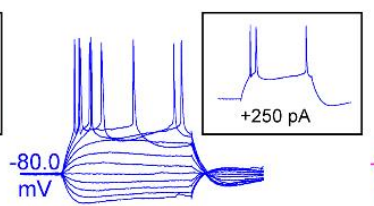

B WT AAV-Control

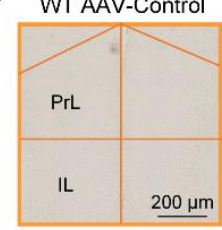

c

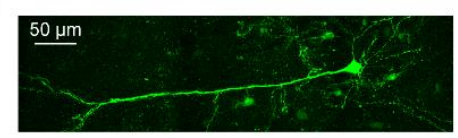

- HOM AAV-FIpO

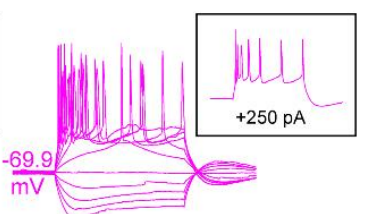

- HOM AAV-FIpO

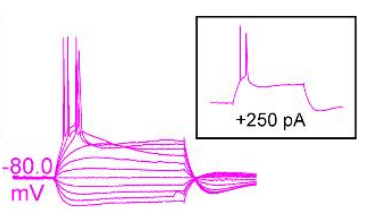

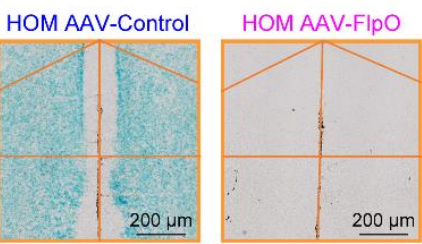

E

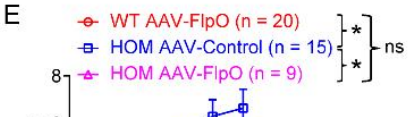

Figure S2. Elevated neuronal firings of layer $\mathrm{V}$ pyramidal cells in the $\mathrm{MPFC}$ are reversible by FlpO-mediated rescue in adult Nav1.2-deficient mice. Related to Figure 2.

(A-B) LacZ staining of coronal brain slices containing mPFC from WT and Scn2agtKo/gtKo (HOM) mice, which were systemically administered with AAV-Control or AAV-FlpO. PrL, prelimbic cortex; IL, infralimbic cortex. (C) A typical layer V pyramidal neuron in the mPFC was labeled by neurobiotin. Scale bar, $50 \mu \mathrm{m}$. (D) Representative current-clamp recordings of pyramidal cells from WT mice transduced with AAV-FlpO (red), HOM mice transduced with AAV-Control (blue), and HOM mice transduced with AAV-Control (magenta) at the RMP. A series of $400-\mathrm{ms}$ hyperpolarizing and depolarizing steps in 50-pA increments were applied to produce the traces. Inset: representative trace in response to $250 \mathrm{pA}$ positive current injection. (E) The average number of APs generated in response to depolarizing current pulses at the RMP. Unpaired twotailed non-parametric Mann-Whitney U-test for each current pulse: ns, no significance, $p>0.05$; ${ }^{*} p<0.05$. (F) Representative current-clamp recordings of layer $\mathrm{V}$ pyramidal cells in the mPFC from WT transduced with AAV-FIpO (red), HOM transduced with AAV-Control (blue) and HOM transduced with AAV-Control (magenta) at a fixed membrane potential of $-80 \mathrm{mV}$. A series of 400-ms hyperpolarizing and depolarizing steps in 50-pA increments were applied to produce the traces. Inset: representative trace in response to $250 \mathrm{pA}$ positive current injection. $(\mathbf{G})$ The average number of APs generated in response to depolarizing current pulses at $-80 \mathrm{mV}$. Unpaired two-tailed non-parametric Mann-Whitney $U$-test for each current pulse: ns, no significance, $p>0.05 ;{ }^{*} p<0.05$. Data were shown as mean \pm SEM. 


\section{Supplementary Figure 3}

A

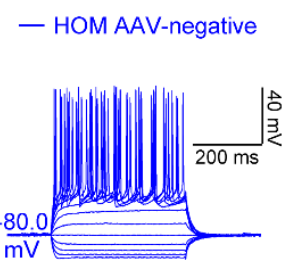

C(i)

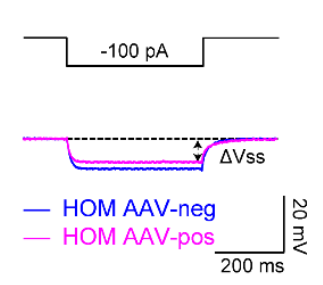

MSNs fixed at a membrane potential of $-80 \mathrm{mV}$
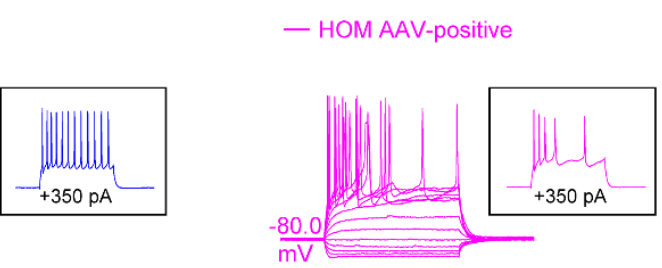

C(ii)

D

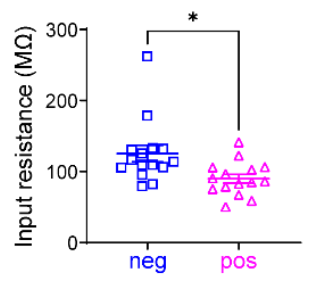

G

F

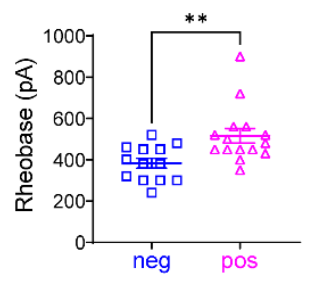

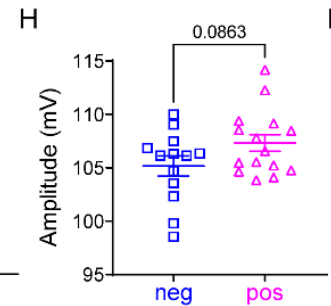

- HOM AAV-neg
- HOM AAV-pos

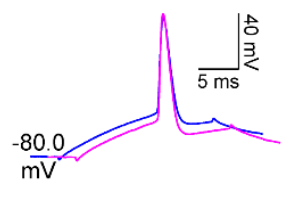

B

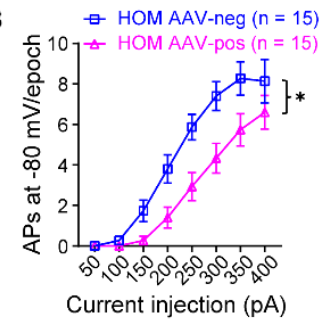

E

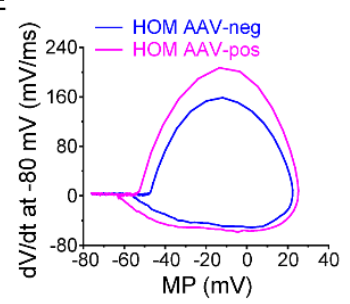

J

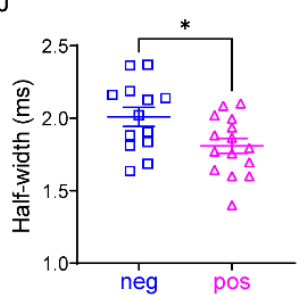

Figure S3. Ex vivo recordings of MSNs at a fixed membrane potential of $-80 \mathrm{mV}$ in adult Nav1.2-deficient mice with a dilute AAV-FIpO-mCherry injection. Related to Figure 3.

(A) Representative current-clamp recordings of MSNs with AAV-negative (blue) and with AAVFlpO-positive (magenta) in Scn2agtKo/gtKo (HOM) mice were obtained at a fixed membrane potential of $-80 \mathrm{mV}$. A series of $400-\mathrm{ms}$ hyperpolarizing and depolarizing steps in 50-pA increments were applied to produce the traces. Inset: representative trace in response to $350 \mathrm{pA}$ positive current injection. (B) The average number of APs generated in response to depolarizing current pulses. Unpaired two-tailed non-parametric Mann-Whitney U-test for each current pulse: ${ }^{*} \mathrm{p}<0.05$. (Ci) Representative traces in response to $100 \mathrm{pA}$ negative current injection. $V_{\text {steady-state }}$ $\left(\mathrm{V}_{\mathrm{ss}}\right)$ is the voltage recorded at $0-10 \mathrm{~ms}$ before the end of the stimulus. (Cii) Individuals and average input resistance values at $-80 \mathrm{mV}$. Unpaired two-tailed Student's $t$-test: ${ }^{*} p<0.05$. (D) Typical spikes of MSNs with AAV-negative (blue) or AAV-FlpO-positive (magenta) in HOM mice were obtained at a fixed membrane potential of $-80 \mathrm{mV}$. (E) Associated phase-plane plots at -80 $\mathrm{mV}$. (F-J) Individuals and average spike rheobase, voltage threshold, amplitude, fAHP, and halfwidth values. Unpaired two-tailed Student's $t$-test: ns, no significance, $p>0.05 ;{ }^{*} p<0.05 ;{ }^{* *} p<$ 0.01. Data were shown as mean \pm SEM. 
bioRxiv preprint doi: https://doi.org/10.1101/2021.02.02 429384 - this version posted February 2, 2021. The copyright holder for this preprint (which was not certified by peer review) is the author/funder, who has granted bioRxiv a license to display the preprint in perpetuity. It is made available under aCC-BY-NC-ND 4.0 International license.

\section{Supplementary Figure 4}

A

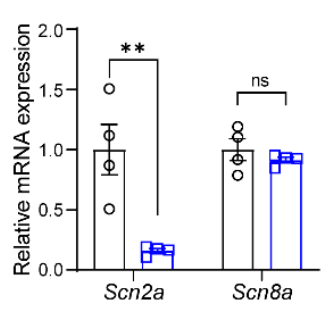

C

- HOM Control

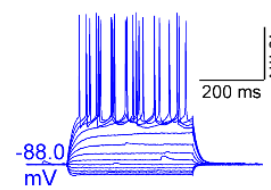

F

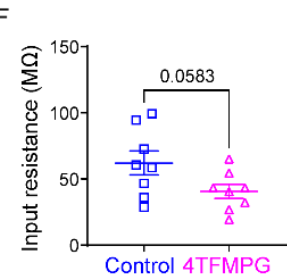

O WT

口 HOM

K L

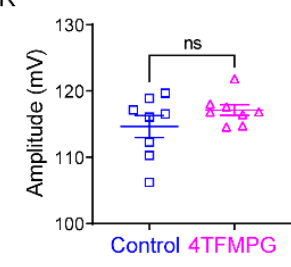

N

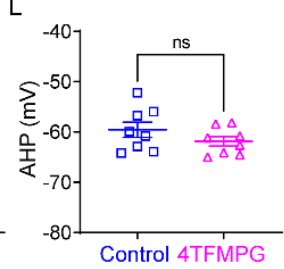

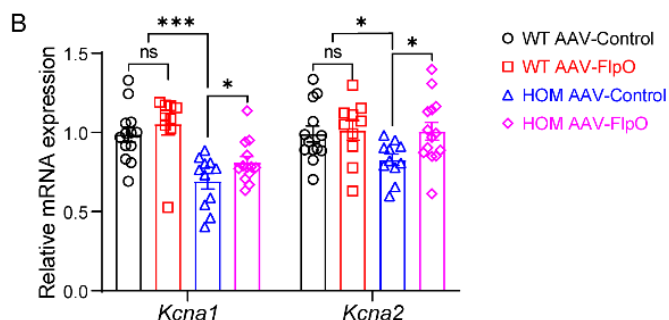

\section{MSNs fixed at the normal RMP}

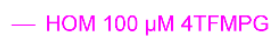
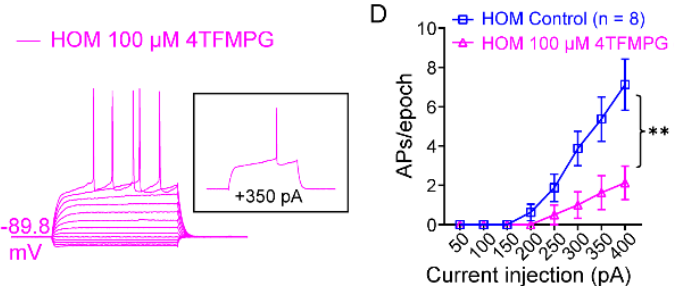

I

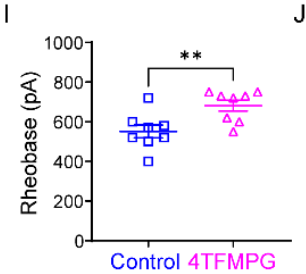

$J$
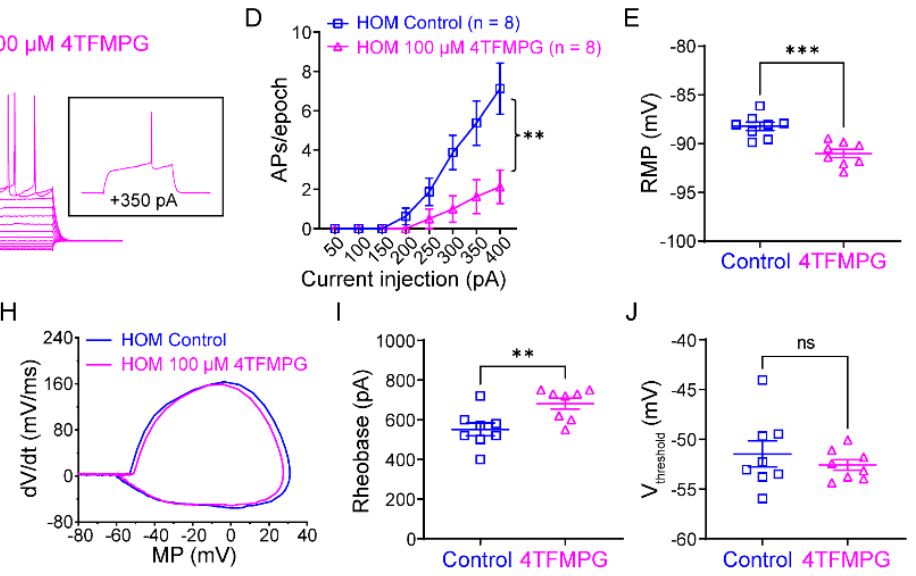

Control 4TFMPG

M

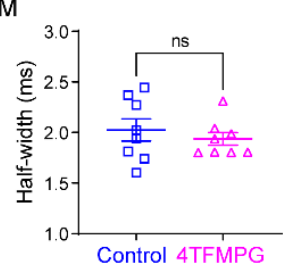

MSNs fixed at a membrane potential of $-80 \mathrm{mV}$

- HOM Control

— HOM $100 \mu \mathrm{M}$ 4TFMPG
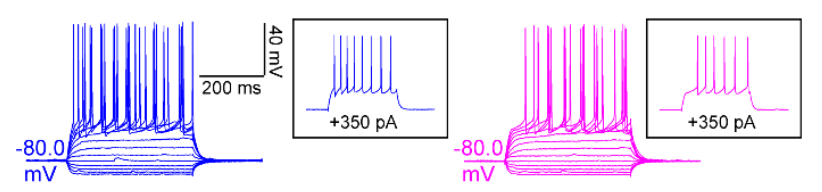

Q

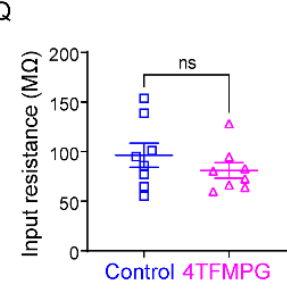

R
- HOM Control
- HOM 100

- HOM Control
- HOM $100 \mu \mathrm{M}$ 4TFMPG

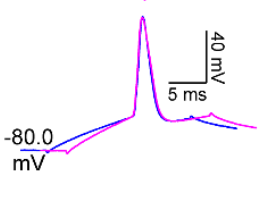

$\mathrm{S}$

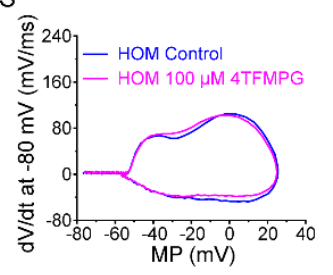

W

V
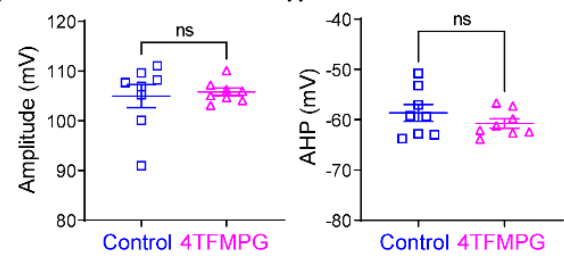

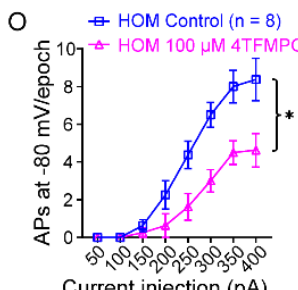

Current injection (pA)

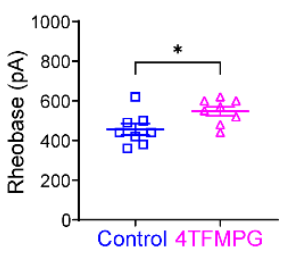

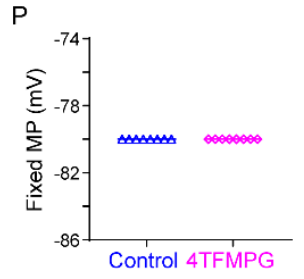

U

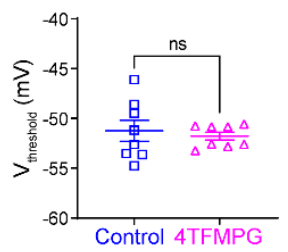

$\mathrm{X}$

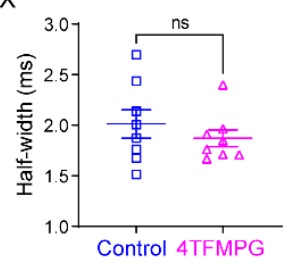


Figure S4. Specific activation of $\mathrm{K}_{\mathrm{v}} 1.1$ channel by 4TFMPG reverses the elevated neuronal firings in adult Nav1.2-deficient mice. Related to Figure 4.

(A) Quantitative (q)PCR analysis of Scn2a and Scn8a mRNA in the striatum samples from WT and Scn2a $a^{\text {tkO/gtKo }}$ mice. Unpaired two-tailed Student's $t$-test for each group: ns, no significance, ${ }^{*} p>0.05 ;{ }^{* *} p<0.01$. (B) qPCR analysis of Kcna1 and Kcna2 mRNA in the striatum samples from WT and HOM mice transduced with AAV-Control or AAV-FIpO, showing that the downregulated mRNA levels of $\mathrm{K}_{v} 1.1$ and $\mathrm{K}_{v} 1.2$ were reversible by FlpO-mediated restoration of Nav1.2 expression in adult Nav1.2-deficient mice. Unpaired two-tailed Student's $t$-test: ns, no significance, ${ }^{*} p>0.05 ;{ }^{*} p<0.05 ;{ }^{* * *} p<0.001$. (C) Representative current-clamp recordings of MSNs from HOM slices perfused with aCSF (HOM Control, blue) and HOM slices perfused with aCSF containing 4TFMPG (HOM $100 \mu \mathrm{M}$ 4TFMPG, magenta) at the RMP. A series of 400-ms hyperpolarizing and depolarizing steps in 50-pA increments were applied to produce the traces. Inset: representative trace in response to $350 \mathrm{pA}$ positive current injection. (D) The average number of APs generated in response to depolarizing current pulses at the RMP. Unpaired twotailed non-parametric Mann-Whitney U-test for each current pulse: ${ }^{* *} p<0.01$. (E) Individuals and average spike RMP values. Unpaired two-tailed Student's $t$-test: ${ }^{* * *} p<0.001$. (F) Individuals and average input resistance values at the RMP. Unpaired two-tailed Student's $t$-test: $p=0.0583$. (G) Typical spikes of MSNs from HOM slices perfused with aCSF (HOM Control, blue) and HOM slices perfused with aCSF containing 4TFMPG (HOM 100 MM 4TFMPG, magenta) were obtained at the RMP. (H) Associated phase-plane plots. (I-M) Individuals and average spike rheobase, voltage threshold, amplitude, fAHP, and half-width values. (N) Representative current-clamp recordings of MSNs from HOM slices perfused with aCSF (HOM Control, blue) and HOM slices perfused with aCSF containing 4TFMPG (HOM $100 \mu \mathrm{M}$ 4TFMPG, magenta) at a fixed membrane potential of $-80 \mathrm{mV}$. A series of $400-\mathrm{ms}$ hyperpolarizing and depolarizing steps in $50-\mathrm{pA}$ increments were applied to produce the traces. Inset: representative trace in response to $350 \mathrm{pA}$ positive current injection. (O) The average number of APs generated in response to depolarizing current pulses at $-80 \mathrm{mV}$. Unpaired two-tailed non-parametric Mann-Whitney $U$-test for each current pulse: ${ }^{*} p<0.05$. (P) fixed MP values for recording. (Q) Individuals and average input resistance values at $-80 \mathrm{mV}$. Unpaired two-tailed Student's $t$-test: $\mathrm{ns}$, no significance, ${ }^{*} \mathrm{p}>0.05$. (R) Typical spikes of MSNs from Scn2a ${ }^{g t K O / g t K O}$ slices perfused with aCSF (HOM Control, blue) and Scn2a $a^{g t K O / g t K O}$ slices perfused with aCSF containing 4TFMPG (HOM $100 \mu \mathrm{M}$ 4TFMPG, magenta) were obtained at a fixed membrane potential of $-80 \mathrm{mV}$. (S) Associated phase-plane plots. (T-X) Individuals and average spike rheobase, voltage threshold, amplitude, fAHP, and halfwidth values. Unpaired two-tailed Student's $t$-test: ns, no significance, ${ }^{*} p>0.05$; ${ }^{*} p<0.05$. Data were shown as mean \pm SEM.

1224

1225

1226 


\section{Supplementary Figure 5}

A

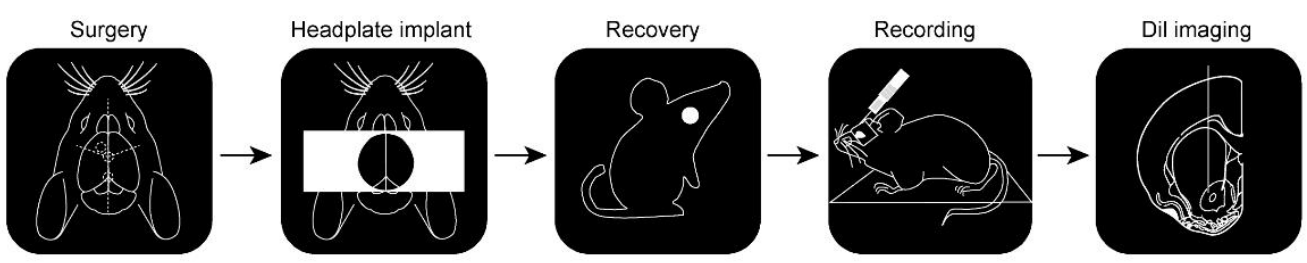

B

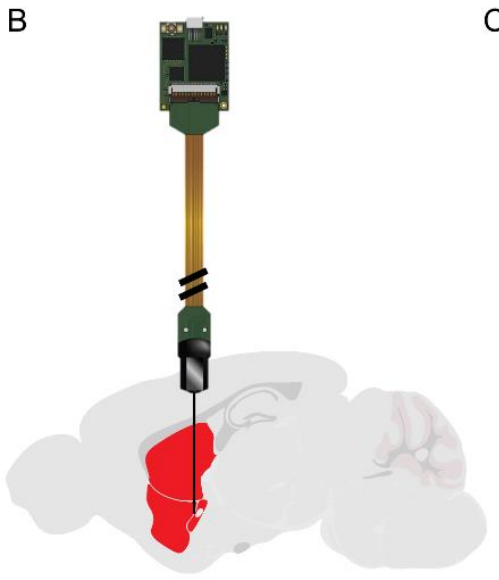

D

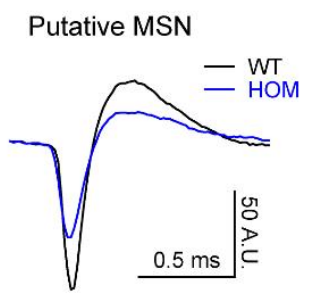

C

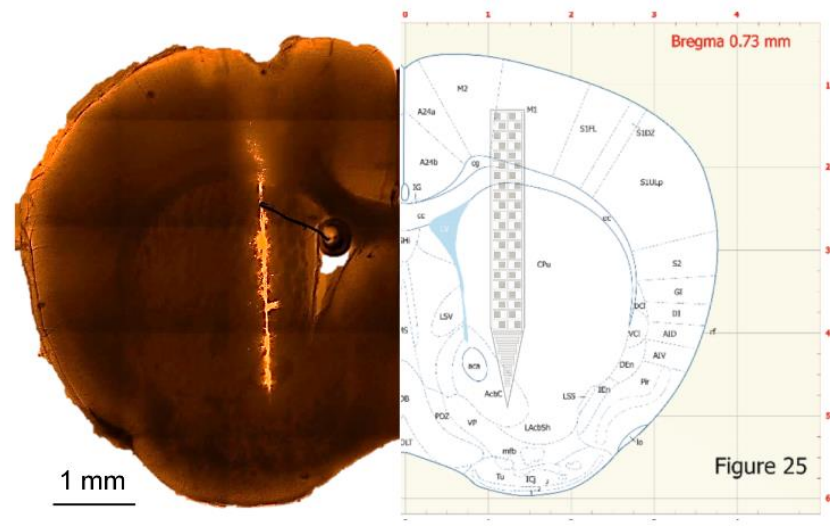

$E(i)$

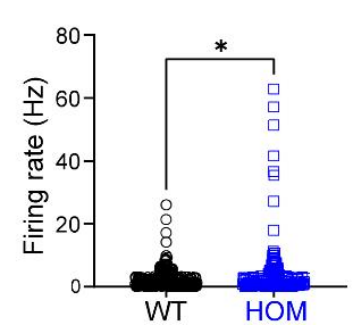

E(ii)

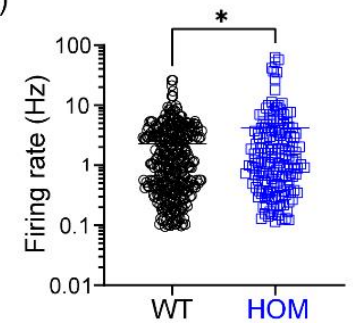

1229

1230

1231

1232

1233

1234

1235

1236

1237

1238

1239

Figure S5. Elevated in vivo neuronal firings of putative striatal MSNs in adult Nav1.2deficient mice.

(A) Cartoon icons showing 5 steps in the Neuropixels recording experiment pipeline. (B) A cartoon illustration of a Neuropixels probe inserted into the striatum. (C) Dil staining of Neuropixels probe after recording in the mouse brain matched with brain map (the right panel was adapted from Figure 25 in the Paxinos and Franklin's The Mouse Brain in Stereotaxic Coordinates). (D) Representative spike waveforms of Neuropixels recordings from putative MSNs of WT (black) and HOM (blue) mice. (Ei): Firing rate of putative MSNs of WT and HOM mice. (Eii): $y$-axis in log scale to show the firing rate of putative MSNs. $n=3$ mice for each genotype; unpaired two-tailed Welch's $t$-test: ${ }^{*} p<0.05$. Data were shown as mean \pm SEM. 